\title{
Budgeting in International Humanitarian Organizations
}

\author{
Milad Keshvari Fard \\ Newcastle University Business School, NE1-4SE Newcastle upon Tyne, United Kingdom. milad.keshvarifard@newcastle.ac.uk \\ Ivana Ljubić \\ Decision Science, ESSEC Business School, 95021 Cergy, France. ivana.ljubic@essec.edu \\ Felix Papier \\ Operations Management, ESSEC Business School, 95021 Cergy, France.papier@essec.edu
}

Problem definition: International humanitarian organizations (IHOs) prepare a detailed annual allocation plan for operations that will be conducted in the countries they serve. The annual plan is strongly affected by the available financial budget. The budget of IHOs is derived from donations, which are typically limited, uncertain, and to a large extent earmarked for specific countries or programs. These factors, together with the specific utility function of IHOs, render budgeting for IHOs a challenging managerial problem. In this paper, we develop an approach to optimize budget allocation plans for each country of operations.

Academic relevance: The current research provides a better understanding of the budgeting problem in IHOs, given the increasing interest of the operations management community for nonprofit operations.

Methodology: We model the problem as a two-stage stochastic optimization model with a concave utility function, and identify a number of analytical properties for the problem. We develop an efficient generalized Benders decomposition algorithm as well as a fast heuristic.

Results: Using data from the International Committee of the Red Cross (ICRC), our results indicate $21.3 \%$ improvement in the IHO's utility by adopting stochastic programming instead of the expected value solution. Moreover, our solution approach is computationally more efficient than other approaches.

Managerial insights: Our analysis highlights the importance of nonearmarked donations for the overall performance of IHOs. We also find that putting pressure on IHOs to fulfill the targeted missions (e.g., by donors or media) results in a lower beneficiaries' welfare. Moreover, the IHOs benefit from negative correlation among donations. Finally, our findings indicate that if donors allow the IHO to allocate unused earmarked donations to other delegations, the performance of IHO improves significantly.

Keywords: International humanitarian organization; Earmarked donations; Budgeting; Nonlinear stochastic programming; Generalized Benders decomposition. 


\section{Introduction}

International humanitarian organizations (IHOs) operate worldwide to run relief operations and development programs. Typically, IHOs have headquarters (HQ) that are responsible for strategic planning and budgeting decisions as well as a number of national delegations that coordinate activities within each country of operation.

In this paper, we study the problem of budgeting in an IHO that is prepared at the HQ level, given the specific characteristics of budget and operations in the humanitarian sector. At the beginning of each year, an IHO typically makes annual plans regarding its operations in each country based on factors such as the humanitarian needs, the ability of the organization to deliver aid, and a realistic assessment of what can actually be implemented. The result is a detailed plan breaking down the required budget based on the country, operations, targeted population, and other factors. However, unlike corporate supply chains (which are financed by revenues from ongoing operations) and entrepreneurial ventures (which are financed from private and corporate venture capital), IHOs typically finance their operations from donations. Relying on donations is challenging, because of the typical lack of sufficient donations, the uncertainties in both the amount and timing of donations, and the fact that part of the financial donations are typically earmarked to specific regions or programs (Van Wassenhove 2006). For example, the International Committee of the Red Cross (ICRC) allows its donors to earmark their donations to a specific country, region, or operation of their choice.

Budget earmarking is one of the main sources of complexity in IHOs. According to Eichenauer and Reinsberg (2017), the amount of bilateral and earmarked aid (both to the NGOs and multilateral agencies) that was donated by OECD members from 1990 to 2012 has increased from USD 50 billion to almost USD 120 billion, while their nonearmarked donations to the multilateral agencies remained almost unchanged in the same period (USD 20 billion). The share of nonearmarked donations received by ICRC has decreased from $29 \%$ to $22 \%$ between 2012 and 2017. Such an increasing trend in the amount of earmarked donations can be a serious challenge to IHOs and has negative impact on humanitarian operations. However, since it reduces the fundraising costs of the IHO (Toyasaki and Wakolbinger 2014) and provides a higher control over the donations 
Keshvari Fard, Ljubić, and Papier: Budgeting in International Humanitarian Organizations

Manufacturing \& Service Operations Management 00(0), pp. 000-000, (C) 0000 INFORMS

by donors, budget earmarking has become inevitable and is pursued by both sides. But donors' preferences over the aid recipient destinations may not always match with the IHO's priorities. For instance, none of the five poorest countries in terms of GDP per capita were among the top ten recipients of bilateral aid from the US or the UK. The same situation holds with the multilateral aid (Malik 2018). Collier and Dollar (2002) estimate that an efficient reallocation of current aid among recipient countries would have a similar impact on poverty reduction as tripling aid budgets under the existing aid allocation. These findings highlight that the nonearmarked budget plays a vital role, as it allows decision makers to finance underfunded delegations.

The main objective of an IHO is to minimize humanitarian suffering and to bring welfare to the affected communities (Holguín-Veras et al. 2013). On the other hand, the range of activities undertaken by the IHOs in one country can consist of a diverse set of missions. For example, the World Health Organization (WHO) performs operations in different areas, such as HIV, maternal and child health, water sanitation hygiene, mental health, and vaccination in the same country. Each of these programs represents different impacts on the welfare of the beneficiaries, and investing one dollar of budget in some programs leads to a higher impact on the beneficiaries' welfare than the others (Keshvari Fard et al. 2019). Given a limited budget, IHOs try to fulfill the missions with the highest impact on the welfare of the society. Therefore, the marginal value of each dollar of donations spent in a country decreases in terms of the resulting beneficiaries' welfare.

Looking at the problem from a larger scale, different delegations may not pose the same level of priority to an IHO. The overall population, ongoing civil wars, higher possibility of natural disasters or outbreaks of contagious diseases, the availability of the infrastructure, the education level and the overall welfare of the society, and media coverage are factors that can result in the greater focus of the IHO's leadership on specific countries and regions. The average cost of operations in different countries, i.e., landed cost, is also not the same. Several factors such as the logistics performance index (LPI), the geographical situation of the country, the distance from warehouses and suppliers, the work conditions, the purchasing power parity, and the availability of skills among the local population also determine the impact of one dollar of donations.

Last but not the least, the amount of future donations depends on the IHO's performance (Hyndman and McDonnell 2009); thus, the budget plans and targeted programs should not be overly 
ambitious such that they cannot be met. Having a low implementation rate (the ratio of the expenditures to the initial budget plans) could have a negative impact on the reputation and credibility of the IHO and subsequently direct donors to other organizations in the future. Therefore, IHOs try to be realistic and precise in their targets. For example, the average implementation rate of the ICRC from 2012 to 2016 exceeded 91\%.

Our contribution We model the budgeting problem as a two-stage nonlinear stochastic program with recourse. We develop an efficient generalized Benders decomposition (GBD) algorithm that outperforms other alternative solutions, namely, the extensive form, and the GBD algorithm where its subproblems are solved by a general-purpose nonlinear solver. We also develop a simple heuristic approach that provides budgeting solutions with small optimality gaps in a short computing time. Both approaches outperform the expected value solution by a large margin, highlighting the need for a stochastic formulation of the problem. We also develop two extensions of the main model; in Section 6, we consider the situation in which the distribution of donations depends on the budget targets, and in Appendix B, we study the possibility of budget adjustments during the year. To evaluate our model, we conduct numerical experiments based on data provided by the International Committee of the Red Cross between 2012 and 2017.

We present several interesting results that could help decision makers in humanitarian organizations. We show that the contribution of nonearmarked donations in the utility of the IHO and welfare of beneficiaries is higher than that of the earmarked donations, and this contribution disparity is more significant for larger IHOs. In our experiments, increasing the $22 \%$ share of nonearmarked donations to $50 \%$ of total donations leads to an increases of $19.9 \%$ of the welfare of beneficiaries.

We also find that the more pressure the public and donors put on the IHO to meet its specified objectives or the more diverse operations in a delegation are, the smaller are the optimal targets and the more the welfare of the beneficiaries is reduced. In our experiments, a $50 \%$ increase of the penalty factor on unmet targets leads to a $3.3 \%$ reduction in budget targets.

Finally, we propose a donation scheme in which donors can earmark their donations for a delegation of their choice; however, they approve that their donation can be allocated to other countries 
Keshvari Fard, Ljubić, and Papier: Budgeting in International Humanitarian Organizations

Manufacturing \& Service Operations Management 00(0), pp. 000-000, (C) 0000 INFORMS

if all the planned operations in their chosen country are fulfilled. Our results indicate that such scheme can significantly enhance the utility of IHOs.

Our work contributes to the current literature in three different ways. First, our paper extends existing research on budgeting in IHOs, because our model combines in a new way donation uncertainty with earmarking and with the concave utility functions of delegations. Second, we develop a model of budget allocation for which we derive managerial insights that help decision makers in IHOs to better plan for the operations to implement. Third, from a technical point of view, we develop an efficient GBD algorithm based on a problem-tailored procedure for derivation of Benders cuts as well as a fast heuristic to derive IHO's budgeting decisions.

\section{Literature review}

Financial donations to nonprofit organizations (NPOs) have been subject of several studies from various disciplines (in this section, we use for precision the general term NPO). Several topics, such as determinants of the amount of donations collected by an NPO (Okten and Weisbrod 2000) or the timing of receiving donations (Petrovits 2006), or the so-called crowding-out mechanism between donations of the government and private sector (Ribar and Wilhelm 2002), have received considerable attention in the literature. In the following, we focus on studies that consider the problem from an operational perspective. For a comprehensive review on challenges faced by NPOs, including budget management, please refer to Anheier and Ben-Ner (2003), Tomasini and Van Wassenhove (2009), Berenguer and Shen (2019), and Besiou and Van Wassenhove (2020).

Several research have considered the impact of budget uncertainty on the performance of an NPO. Natarajan and Swaminathan (2014) analyze the impact of uncertainty in the timing of receiving the pledged donations and conclude that the front-loading has a considerable impact on the performance of the NPO. They also show that the benefits of front-loading are significantly lower for products with a low critical ratio. Aflaki and Pedraza-Martinez (2016) show that increasing nonearmarked donations can mitigate the impact of donation uncertainty and improve the NPO's operational efficiency. Devalkar et al. (2017) argues that when a proportion of donors only contribute after the delivery of results, the NPO can use an ex-post funding scheme to reduce the impact of budget uncertainty. Keshvari Fard et al. (2019) show that under a higher budget uncertainty, the deprivation cost increases, while the fleet utilization rate decreases. 
Considering the impact of earmarking, Jahre and Heigh (2008) find that earmarked funding could impair postponement strategies, which are vital for relief operations. Wakolbinger et al. (2011) state that not only the total amount of funds but also speed and timing, flexibility, predictability and fluctuation of funds impact the value of funds from the perspective of an NPO. Pedraza-Martinez and Van Wassenhove (2013) find that incentive issues resulting from earmarked funding increase the operating cost of vehicles in humanitarian operations, because earmarked funding creates incentive misalignment between the NPO's headquarters and the field offices. Besiou et al. (2014) show that earmarked funding decreases the performance of NPOs during disasters, especially if decision making on the vehicle procurement is decentralized. Toyasaki and Wakolbinger (2014) find that although allowing for earmarked donations decreases the fundraising costs and usually results in higher total donations collected, it restricts the efficiency of the NPOs. Bhattacharya et al. (2014) show that even collaboration of different programs in sharing their resources does not necessarily eliminate the inefficiencies associated with earmarked funding.

Accepting earmarked funding as inevitable, a number of studies have designed mechanisms to improve the operations when donors can earmark their donations. Toyasaki and Wakolbinger (2014) consider the reputation of the NPO as well as the media coverage on a disaster the determining factors for the NPOs when deciding upon their fundraising strategies. They conclude that wellknown NPOs should be careful about accepting earmarked donations. Bhattacharya et al. (2014) study the reallocation of earmarked funding between the NPO's programs and show that allowing aid programs the flexibility of transferring primary resources improves the efficiency of the system.

Other studies have shown that lack of coordination among donors, and higher fragmentation of donations, donors and aid channels lead to higher transaction costs and lower operational efficiency of an NPO (Buse and Walt 1996, Acharya et al. 2006, Berenguer et al. 2016).

Finally, several research contributions propose measures to increase the amount of donations, e.g., announcing the past contributions of other donors (Varian 1994), using a publicized and tiered fundraising scheme (McCardle et al. 2009), dynamically allocating assets between revenue generating activities and nonprofit operations (De Vericourt and Lobo 2009), allowing donors to audit and penalize NPOs (Privett and Erhun 2011), and using ex-post funding scheme which allows donors to contribute only after the delivery of NPO's projects (Devalkar et al. 2017). 
Keshvari Fard, Ljubić, and Papier: Budgeting in International Humanitarian Organizations

Manufacturing \& Service Operations Management 00(0), pp. 000-000, (C) 0000 INFORMS

Simpler variants of our model have been studied in the literature. If the utility functions of the delegations were strictly linear, the model would be equivalent to the multiproduct newsvendor problem with uncertain budget and could be solved by the method proposed in Bansal and Dyer (2017). If the budget constraint were also deterministic, the model would further simplify to the well-known multiple newsvendor problem (see, e.g., Erlebacher (2000)). Finally, nonlinear utility functions for a single newsvendor have been studied by Halman et al. (2012).

\section{Model Description and Analytical Results}

In this section, we describe the model and provide some analytical insights into the optimal solution.

\subsection{Model Description}

Consider an IHO that runs operations in $n$ delegations. Let $I=\{1, \ldots, n\}$ be the index set of all delegations. At the beginning of the year, the HQ decides upon the level of operations to be done in each delegation $i \in I$, and consequently the required budget target for this delegation, denoted by $x_{i}$. To finance this target, the HQ first relies on the donations earmarked for that delegation, which are referred to as $\xi_{i} \geq 0$, for each $i \in I$. The value of $\xi_{i}$ is not known at the time of planning; however, the IHO knows its distribution. We denote the marginal probability distribution function of $\xi_{i}$ by $B_{i}\left(\xi_{i}\right)$ and the marginal cumulative distribution function by $\mathbb{B}_{i}\left(\xi_{i}\right)$. The donation distributions $\mathbb{B}_{i}\left(\xi_{i}\right)$ are independent of targets $x_{i}$. In Section 6 we also investigate a setting in which the distribution $\mathbb{B}_{i}\left(\xi_{i}\right)$ depends on $x_{i}$. Note that we do not make any assumption about the correlation among the donation distributions.

If the amount of earmarked donations received for the delegation $i$ is not sufficient to cover all the planned missions, the HQ may allocate part of the nonearmarked budget to the delegation, denoted by $y_{i}$. We denote the part of the nonearmarked donations that can be distributed among delegations, e.g., after deducting the HQ's budget, by $\xi_{n e} \geq 0$ and its density function by $B_{n e}\left(\xi_{n e}\right)$.

For simplicity, we assume that the decision regarding the allocation of nonearmarked budget among the delegations is taken at the end of the year, although technically, the money could be transferred to the delegations in one or more payments at any time of the year, based on the needs of the delegations. For example if the earmarked donations for delegation $i$ are mainly back-loaded, the HQ may allocate some nonearmarked budget to the delegation and receive it back in the future 
when the earmarked donations for that delegation arrive. Such an assumption especially fits with the reality when the arrival of the donations mainly follows a front-loaded pattern, i.e., the majority of the donations arrive early in the period (Natarajan and Swaminathan 2014).

At the beginning of the period, the IHO decides on the budget targets, with the knowledge of the distribution of donations. During the period, the delegations receive the earmarked donations, which are usually immediately available to be allocated to different missions. At the end of the period, given the declared budget targets and the donations received during the period, the IHO finalizes the allocation of the nonearmarked donations, such that the utility is maximized. In Appendix B, we also develop a model in which the IHO can revise its targets during the year.

Note that we only consider two types of donations in this research: earmarked for a delegation, or nonearmarked at all. In reality, however, donors may be given more options to earmark their donations (e.g., earmarking the donation for a program or for a program in a specific country). Nonetheless, reducing the donation patterns to these two schemes is not confining, since most of the donations usually belong to one of the two categories considered in this paper. For example, according to ICRC, from 2015 to 2016 , on average $79 \%$ of the donations were either earmarked for a country or not earmarked at all. Other earmarking schemes can be fitted in the current model with a good approximation.

We assume that even if $\xi_{i}$ exceeds $x_{i}$, the effective budget that will be spent by delegation $i$ is $x_{i}$. This assumption matches the practice of operations in IHOs because fulfilling extra missions usually requires advance planning, extra skills, operational assets, coordination, human resources, and other acticities, and receiving extra budget late in the year usually does not trigger additional operations; instead, the additional budget can be saved for the next year. Therefore, the total effective budget that contributes to the welfare of the beneficiaries in delegation $i$ is identified as $\alpha_{i}=\min \left\{x_{i}, \xi_{i}+y_{i}\right\}$

We consider $f_{i}\left(\alpha_{i}\right)$ to be the resulting beneficiaries' welfare from spending a budget of $\alpha_{i}$ in delegation $i$. We assume $f_{i}, i \in I$ to be strictly increasing, concave, and twice differentiable, i.e., allocating extra budget results in fulfilling additional missions and therefore higher welfare for the beneficiaries; however, the marginal impact of these additional missions on the beneficiaries' welfare 
is decreasing. If the allocated budget to delegation $i, \xi_{i}+y_{i}$, is less than $x_{i}$, the IHO fails to fulfill the planned missions, which brings disutility for the IHO. This disutility can be modeled as a function of the budget shortage for delegation $i \in I$, defined as $\left[x_{i}-\xi_{i}-y_{i}\right]^{+}$, where $[x]^{+}=\max \{0, x\}$. We refer to this disutility as $g_{i}\left(\left[x_{i}-\xi_{i}-y_{i}\right]^{+}\right)$, which can be simplified to $g_{i}\left(x_{i}-\alpha_{i}\right)$. Since $g_{i}$ is a loss function, it should be increasing and convex in its argument. For simplicity, in this paper we assume that $g_{i}$ is linear for all $i \in I$, i.e., $g_{i}()=.a_{i}^{g}[.]^{+}, a_{i}^{g} \geq 0$, even though our GBD method and most of our analytical findings also apply to convex, nonlinear $g_{i}$ functions. Finally, we refer to the net utility for delegation $i$, given $x_{i}, y_{i}$ and $\xi_{i}$ as the following:

$$
u_{i}\left(x_{i}, \xi_{i}, y_{i}\right)=f_{i}\left(\min \left\{x_{i}, \xi_{i}+y_{i}\right\}\right)-g_{i}\left(\left[x_{i}-\xi_{i}-y_{i}\right]^{+}\right), \quad i \in I
$$

Hence, the overall goal of the IHO is to decide the target values of $x_{i} \geq 0, i \in I$, so that the sum of the expected net utilities over all delegations is maximized. Referring to the budget targets as $\boldsymbol{x}=\left[x_{1}, x_{2}, \ldots, x_{n}\right]$, the donations received by the IHO as $\boldsymbol{\xi}=\left[\xi_{1}, \xi_{2}, \ldots, \xi_{n}, \xi_{n e}\right]$ and the allocation of the nonearmarked budget to different delegations as $\boldsymbol{y}=\left[y_{1}, y_{2}, \ldots, y_{n}\right]$, we can formulate the budget allocation problem as the following two-stage stochastic problem:

$$
\text { Stage 1: } \quad \max _{\boldsymbol{x} \geq 0} \mathfrak{Q}(\boldsymbol{x})=\mathbb{E}_{\tilde{\boldsymbol{\xi}}}[Q(\boldsymbol{x}, \boldsymbol{\xi})]
$$

Stage 2: $\quad Q(\boldsymbol{x}, \boldsymbol{\xi})=\max _{\boldsymbol{y} \geq 0} U(\boldsymbol{x}, \boldsymbol{\xi}, \boldsymbol{y})$

$$
\text { s.t. } \quad \sum_{i=1}^{n} y_{i} \leq \xi_{n e},
$$

where $\boldsymbol{\xi}$ refers to the vector of random variables modeling uncertain donations, and $U(\boldsymbol{x}, \boldsymbol{\xi}, \boldsymbol{y})=$ $\sum_{i=1}^{n} u_{i}\left(x_{i}, \xi_{i}, y_{i}\right)$ calculates the overall utility under budget targets of $\boldsymbol{x}$, observed donations of $\boldsymbol{\xi}$, and allocation of nonearmarked donations of $\boldsymbol{y} . Q(\boldsymbol{x}, \boldsymbol{\xi})$ is called the recourse function and refers to the maximum possible utility for a given $\boldsymbol{x}$ and $\boldsymbol{\xi}$, and finally $\mathfrak{Q}(\boldsymbol{x})$ is the expected overall utility that the IHO can obtain under a given $\boldsymbol{x}$.

Lemma 1identifies the behavior of the allocation decisions in optimality. All proofs and additional technical results are contained in the Supplementary Appendix, which is available online at https: //papers.ssrn.com/sol3/papers.cfm?abstract_id=3464619.

Lemma 1. Given $\boldsymbol{x}$ and $\boldsymbol{\xi}$, for the optimal allocation decisions, $\boldsymbol{y}^{*}$, we have $y_{i}^{*} \leq\left[x_{i}-\xi_{i}\right]^{+}$, for all $i \in I$. 
Given Lemma 1, we propose a simplified formulation of the recourse function $Q(\boldsymbol{x}, \boldsymbol{\xi})$, by adding the constraints $y_{i} \leq\left[x_{i}-\xi_{i}\right]^{+}, i=\{1, \ldots, n\}$ to the model which leads to the following:

$$
\begin{aligned}
Q(\boldsymbol{x}, \boldsymbol{\xi})=\max _{\boldsymbol{y} \geq \mathbf{0}} U(\boldsymbol{x}, \boldsymbol{\xi}, \boldsymbol{y})= & \max _{\boldsymbol{y} \geq \mathbf{0}} \sum_{i \in \mathfrak{C}(\boldsymbol{x}, \boldsymbol{\xi})}\left[f_{i}\left(\xi_{i}+y_{i}\right)-g_{i}\left(x_{i}-\xi_{i}-y_{i}\right)\right]+\sum_{i \notin \mathfrak{C}(\boldsymbol{x}, \boldsymbol{\xi})} f_{i}\left(x_{i}\right), \\
\text { s.t. } \quad y_{i} \leq x_{i}-\xi_{i}, \quad i \in \mathfrak{C}(\boldsymbol{x}, \boldsymbol{\xi}) & \\
& \sum_{i \in \mathfrak{C}(\boldsymbol{x}, \boldsymbol{\xi})} y_{i} \leq \xi_{n e},
\end{aligned}
$$

where $\mathfrak{C}(\boldsymbol{x}, \boldsymbol{\xi})=\left\{i \in I: x_{i} \geq \xi_{i}\right\}$ refers to the set of delegations that can benefit from the nonearmarked donations, i.e., for which $y_{i} \geq 0$.

\subsection{Analytical insights into the optimal solution}

We begin by analyzing the role of earmarked donations. Proposition 1 compares the importance of these donations to nonearmarked donations for the IHOs' utility and the welfare of beneficiaries.

Proposition 1. Regarding the earmarked and nonearmarked donations, we have the following:

1. $\frac{\partial Q}{\partial \xi_{n e}}(\boldsymbol{x}, \boldsymbol{\xi}) \geq \frac{\partial Q}{\partial \xi_{i}}(\boldsymbol{x}, \boldsymbol{\xi})$, for all $i \in I$, and at any given point $(\boldsymbol{x}, \boldsymbol{\xi}) \geq \mathbf{0}$.

2. $\frac{\partial Q}{\partial \xi_{n e}}$ is non decreasing in $n$.

3. With $f_{i}\left(\alpha_{i}\right)=a_{i}^{f}\left(\alpha_{i}\right)^{b_{i}^{f}}, a_{i}^{f} \geq 0$, and $0<b_{i}^{f} \leq 1, \frac{\partial Q / \partial \xi_{i}}{\partial Q / \partial \xi_{n e}}$ is non decreasing in $a_{i}^{f}$, $b_{i}^{f}$ and $a_{i}^{g}$ for all $i \in I$.

4. The contribution of nonearmarked donations to the IHO's overall utility are higher than that of earmarked donations.

Part 1 of the proposition states that the contribution of one dollar of nonearmarked donations to the IHOs' utility in the recourse function is higher than that of the earmarked donations, which is intuitive because nonearmarked donations have less constraints on their use. Part 2 states that by expanding the operations to a larger number of delegations, it becomes more important for the IHO to receive more nonearmarked donations. Part 3 implies that while nonearmarked donations are always preferred, for delegations with higher importance (larger $a_{i}^{f}$, e.g., higher urgency of missions, larger population of beneficiaries), higher concentration of operations (larger $b_{i}^{f}$ ), or larger media attention (larger $a_{i}^{g}$ ), earmarked donations have higher contributions than those for delegations that do not meet these conditions. Part 4 extends the results to the overall IHO utility, $\mathfrak{Q}\left(\boldsymbol{x}^{*}\right)$. 
It is straightforward to show that Proposition 1 holds true if the IHO's utility $\sum_{i=1}^{n} u_{i}\left(x_{i}, \xi_{i}, y_{i}\right)$ is replaced with the overall beneficiaries' welfare, $\sum_{i=1}^{n} f_{i}\left(\alpha_{i}\right)$. In other words, the aforementioned advantages of earmarked donations are still valid even if the IHO focuses exclusively on the maximization of the beneficiaries' welfare. Note that in such a case, there would be no finite solution to the problem, and the IHO would set $\boldsymbol{x}=\infty$.

Next, we show how different schemes of implementing a more flexible budget allocation affect the IHO utility. We define a partially relaxed earmarking scheme as the setting that allows the IHO to re-allocate the surplus of funds for a delegation $i,\left[\xi_{i}-x_{i}\right]^{+}$. In other words, donations earmarked for $i$ that exceed the target of delegation $i$ can be allocated to other delegations. Let us define $Q^{\prime}$ as the recourse function of this scheme, which can be modeled by replacing the constraint of the second stage in Model (1) with $\sum_{i=1}^{n} y_{i} \leq \xi_{n e}+\sum_{i=1}^{n}\left[\xi_{i}-x_{i}\right]^{+}$. We compare this scheme against a full relaxation of the earmarking constraints, which we refer to as fully relaxed earmarking scheme. Let us denote its recourse function by $Q^{\prime \prime}$. The scheme can be modeled by replacing the non-negativity of $y_{i}$ with $y_{i} \geq-\xi_{i}$ for all $i$, in Model (1). Corollary 1 shows the order of the IHO utility under the different schemes.

\section{Corollary 1.}

$$
\mathfrak{Q}\left(\boldsymbol{x}^{*}\right) \leq \max _{\boldsymbol{x} \geq \mathbf{0}} \mathbb{E}_{\tilde{\boldsymbol{\xi}}}\left[Q^{\prime}(\boldsymbol{x}, \boldsymbol{\xi})\right] \leq \max _{\boldsymbol{x} \geq \mathbf{0}} \mathbb{E}_{\tilde{\boldsymbol{\xi}}}\left[Q^{\prime \prime}(\boldsymbol{x}, \boldsymbol{\xi})\right]
$$

This finding highlights the importance of a partial earmarking scheme for an IHO, which we analyze in detail in Section 6.

Proposition 2 analyzes the sensitivity of the budget target with respect to the model parameters.

Proposition 2. With $f_{i}\left(\alpha_{i}\right)=a_{i}^{f}\left(\alpha_{i}\right)^{b_{i}^{f}}, x_{i}^{*}$ is non decreasing in $a_{i}^{f}$ and $b_{i}^{f}$ and non increasing in $a_{i}^{g}$.

Proposition 2 implies that for delegations with higher importance and urgency of missions or delegations with a less diverse (e.g., type, location, target groups) set of operations, a higher budget target should be set. This is interesting, because it implies that delegations with more diverse operations receive lower targets. On the other hand, if the penalty of target fulfillment for a delegation is large, the IHO should act conservatively and set a lower budget target.

It is also straightforward to infer that in optimality, the overall beneficiaries' welfare $\sum_{i=1}^{n} f_{i}\left(\alpha_{i}\right)$ is decreasing in $\boldsymbol{a}^{g}=\left[a_{1}^{g}, \ldots, a_{n}^{g}\right]$. In other words, with a lower pressure on the IHO to meet its targets (e.g., by media), the contribution of the IHO to the society is higher. 
Even though the current research focuses on IHOs, the budgeting problem may exist in local humanitarian organizations as well. Proposition 3 determines the budget target for a single delegation (in which case all donations can be considered as earmarked).

Proposition 3. If $n=1$ the optimal budget target $x^{*}$ is given by the following:

$$
x^{*}=\mathbb{B}^{-1}\left(\frac{f^{\prime}(x)}{f^{\prime}(x)+a^{g}}\right),
$$

where $f^{\prime}(x)$ is the derivative of $f(x), \mathbb{B}($.$) is the cumulative distribution function of the earmarked$ donations received by the humanitarian organization, and $\mathbb{B}^{-1}($.$) its inverse function.$

Note that if function $f$ is linear in its argument, then the solution of Proposition 3 reduces to the well-known newsvendor solution.

\section{Solution Methods}

In this section we propose three ways to approach the problem from a computational perspective. The first two methods are based on sampling a finite number of possible scenario realizations and solving the underlying problem approximation. They provide exact solutions for the given subset of scenarios. The first one (cf. Section 4.1) is based on a deterministic reformulation of the problem as a nonlinear program. Due to the size of the model and the nonlinearity of the objective function, its application is limited to small problem inputs. However, this deterministic reformulation provides theoretical foundation for deriving a much efficient exact method, called the generalized Benders decomposition approach, which we present in Section 4.2. The third method (cf. Section 4.3) is a fast and efficient heuristic procedure that exploits the cumulative distribution function of earmarked donations and does not require scenario sampling. This heuristic can be applied (complementary or alternatively to GBD) to large problem inputs to find a high-quality solution in short computing time.

\subsection{Deterministic Equivalent}

In the Model (1), we assume that the donations can be modeled as a random vector $\tilde{\xi}$ with a known probability distribution. To solve Model (1) using optimization techniques, we can assume that $\tilde{\xi}$ has a finite number of possible realizations, called scenarios. In that case, the expectation function can be written as the weighted sum of the recourse values over all possible realizations for the value of $\tilde{\boldsymbol{\xi}}$. In case $\tilde{\boldsymbol{\xi}}$ follows a continuous distribution, we have to discretize its elements. 
Consider a finite set $K$ of different scenarios, with $\boldsymbol{\xi}^{k}$ being the $\mathrm{k}^{\text {th }}$ realization of donations vector $\boldsymbol{\xi}$, with respective probability mass $p_{k}$, for all $k \in K$. In this case, Model (1) can be expressed by its deterministic equivalent, which is a large nonlinear programming problem. The optimal value of the deterministic equivalent is calculated as follows:

$$
\overline{\mathfrak{Q}}^{*}=\max _{\boldsymbol{x} \geq \mathbf{0}} \sum_{k \in K} p_{k} Q\left(\boldsymbol{x}, \boldsymbol{\xi}^{k}\right)
$$

It is well-known that $\overline{\mathfrak{Q}}^{*}$ and the associated optimal solution, $\overline{\boldsymbol{x}}^{*}$, converge to the optimal value $\mathfrak{Q}\left(\boldsymbol{x}^{*}\right)$ and the optimal solution $\boldsymbol{x}^{*}$ of the true problem $\max _{\boldsymbol{x} \geq \mathbf{0}} \mathbb{E}_{\tilde{\boldsymbol{\xi}}}[Q(\boldsymbol{x}, \boldsymbol{\xi})]$, respectively, as $|K| \rightarrow$ $\infty$. Hereinafter, we refer to $\overline{\boldsymbol{x}}^{*}$ and $\overline{\mathfrak{Q}}^{*}$ as the optimal solution and the optimal value of the discretized problem, respectively.

For small $n$ and $|K|$, we can write the deterministic equivalent of the problem and use one of the off-the-shelf nonlinear programming solvers to obtain its optimal solution. Model (6) identifies the extensive form of the deterministic equivalent, in which, for each scenario $k \in K$, we introduce a second-stage variable $\boldsymbol{y}^{k}$. Furthermore, we also introduce variables $\alpha_{i}^{k}$ to linearize the expression $\min \left\{x_{i}, \xi_{i}^{k}+y_{i}\right\}$, for all $i \in I, k \in K$ :

$$
\begin{aligned}
& \max _{\boldsymbol{x}, \boldsymbol{\alpha}, \boldsymbol{y}} \overline{\mathfrak{Q}}(\boldsymbol{x}, \boldsymbol{\alpha}, \boldsymbol{y})=\sum_{k \in K} p_{k} \sum_{i=1}^{n}\left[f_{i}\left(\alpha_{i}^{k}\right)-g_{i}\left(x_{i}-\alpha_{i}^{k}\right)\right] \\
& \text { s.t. } \quad \alpha_{i}^{k} \leq x_{i} \quad k \in K, i \in I \\
& \alpha_{i}^{k} \leq \xi_{i}^{k}+y_{i}^{k} \quad k \in K, i \in I \\
& \sum_{i=1}^{n} y_{i}^{k} \leq \xi_{n e}^{k} \quad k \in K \\
& x_{i}, y_{i}^{k}, \alpha_{i}^{k} \in \mathbb{R}^{+} \quad k \in K, i \in I
\end{aligned}
$$

In this model, $\overline{\mathfrak{Q}}(\boldsymbol{x}, \boldsymbol{\alpha}, \boldsymbol{y})$ denotes the objective function of the discretized problem, $\xi_{i}^{k}$ represents the $\mathrm{k}^{\text {th }}$ realizations of $\tilde{\xi}_{i}, y_{i}^{k}$ is the allocation of $\xi_{n e}^{k}$ to delegation $i$ for realization $k$, and $\alpha_{i}^{k}$ is the effective budget for delegation $i$ under $\boldsymbol{\xi}^{k}$.

Lemma 2. In Model ([6), $\overline{\mathfrak{Q}}(\boldsymbol{x}, \boldsymbol{\alpha}, \boldsymbol{y})$ is jointly concave in $(\boldsymbol{x}, \boldsymbol{\alpha}, \boldsymbol{y})$.

Given Lemma 2, the global optimum can be found for Model (6). We note that the total number of variables and constraints is $O(n \cdot|K|)$; hence, by increasing the number of delegations or the number of scenarios, Model (6) easily becomes numerically intractable. 


\subsection{Generalized Benders Decomposition}

To solve larger instances of the deterministic equivalent, we propose an efficient decomposition method, namely, a generalization of the L-Shaped technique for nonlinear problems, also known as generalized Benders decomposition. In this paper, we design a problem-tailored GBD approach that in contrast to the standard recipes from the literature, does not require a general-purpose nonlinear solver for derivation of Benders cuts and uses a combinatorial procedure instead. For a general introduction to the L-shaped method and (generalized) Benders decomposition, see, e.g., Benders (1962). Recent advances in Benders decomposition for solving mixed-integer (stochastic) optimization problems are presented in Carøe and Tind (1998), Liu et al. (2009), Fischetti et al. (2016, 2017). The concept has been extended to robust optimization (Takriti and Ahmed 2004).

For a given set of scenarios $K$, we project out second-stage decision variables (namely, $\boldsymbol{y}$ and $\boldsymbol{\alpha}$ ), and formulate the whole problem in the natural space of first-stage variables $\boldsymbol{x} \geq 0$. Thereby, we reduce the number of decision variables from $O(n \cdot|K|)$ to $O(n+|K|)$. To this end, instead of solving a compact but nonlinear optimization problem with a large number of variables and constraints given by Model (6), we first reformulate the deterministic equivalent using the value function reformulation as follows:

$$
\begin{aligned}
\max & \sum_{k \in K} p_{k} \theta^{k} \\
& \theta^{k} \leq Q\left(\boldsymbol{x}, \boldsymbol{\xi}^{k}\right), \quad k \in K \\
& \boldsymbol{x} \geq \mathbf{0}, \boldsymbol{\theta} \in \mathbb{R}^{|K|},
\end{aligned}
$$

where $\theta^{k}$ refers to the underestimation of the overall utility under $\boldsymbol{\xi}^{k}$. Due to the maximization of the objective function, at optimality, equality is attained.

Since we are dealing with a problem with a complete recourse, the problem $Q\left(\boldsymbol{x}, \boldsymbol{\xi}^{k}\right)$ is well defined for any $\boldsymbol{x} \geq \mathbf{0}$ and any $\boldsymbol{\xi}^{k}$. To obtain the Benders reformulation, we will replace constraints (8) with a family of linear Benders optimality constraints. These cuts are separated on the fly, in a cutting plane fashion. The relaxed Benders master problem contains only a small fraction of these cuts (at the beginning, it can be empty), and whenever an optimal solution $(\check{\boldsymbol{x}}, \check{\boldsymbol{\theta}}$ ) of the relaxed master problem is found, its feasibility is validated by solving the Benders subproblem. The latter 
consists of checking whether $\theta^{k} \leq Q\left(\check{\boldsymbol{x}}, \boldsymbol{\xi}^{k}\right)$, for all $k \in K$. If this is not the case, a violated Benders cut is detected, inserted into the master problem, and the process is repeated until $\epsilon$-convergence is achieved. We observe that in this process, we need to solve Benders subproblems for all $|K|$ realizations of $\boldsymbol{\xi}$. Given that the subproblems are nonlinear and require considerable computational efforts, it is better to extract as much information as possible at each iteration by adding multiple cuts each time the Benders subproblem is called. This leads to the multicut approach (see, e.g., Birge and Louveaux (2011), You and Grossmann (2013), Leitner et al. (2018)).

In the following, we provide a closed-form formula to calculate Benders optimality cuts for any given solution of the master problem $(\check{\boldsymbol{x}}, \check{\boldsymbol{\theta}})$. We first observe that the function $Q\left(\check{\boldsymbol{x}}, \boldsymbol{\xi}^{k}\right)$ is concave in $\boldsymbol{x}$, that the Slater conditions hold and that the maximum is attained (cf. Lemma 3 in the supplementary appendix). Let $Q\left(\check{\boldsymbol{x}}, \boldsymbol{\xi}^{k}\right)$ denote the optimal solution value of the $k$-th Benders subproblem, and let $\check{\boldsymbol{y}}^{k}$ be the corresponding optimal solution. If $\tilde{\theta}^{k}>Q\left(\check{\boldsymbol{x}}, \boldsymbol{\xi}^{k}\right)$, a violated Benders cut for the $k$-th subproblem can be obtained by overestimating the value of $Q\left(\boldsymbol{x}, \boldsymbol{\xi}^{k}\right)$ by a supporting hyperplane in $\check{\boldsymbol{x}}$ as follows:

$$
\theta^{k} \leq Q\left(\check{\boldsymbol{x}}, \boldsymbol{\xi}^{k}\right)+\boldsymbol{\lambda}^{k}(\boldsymbol{x}-\check{\boldsymbol{x}})
$$

where $\boldsymbol{\lambda}^{k}$ denotes a supergradient of the concave function $Q\left(\boldsymbol{x}, \boldsymbol{\xi}^{k}\right)$ at point $\check{\boldsymbol{x}}$. Rather than having these supergradients computed from a black-box nonlinear solver (using the tools of convex optimization), Theorem 1 shows how to obtain the subgradients in a combinatorial fashion.

Deriving a Benders cut (9) requires solving a deterministic convex allocation problem, for which there is a vast literature on solution methods (see, e.g., Patriksson (2008) for an overview). In our implementation, due to the simplicity of our allocation model, we use a modified version of Zipkin (1980)'s algorithm to find the optimal solution for $Q\left(\check{\boldsymbol{x}}, \boldsymbol{\xi}^{k}\right)$. Once this solution is attained, the Benders cut can be derived according to the result shown below.

Theorem 1. Let $\check{\boldsymbol{y}}$ denote the optimal solution of the convex allocation problem $Q\left(\check{\boldsymbol{x}}, \boldsymbol{\xi}^{k}\right)$. Coefficients of the supergradient $\boldsymbol{\lambda}^{k}$ in the Benders optimality cut (9) can be calculated as follows:

$$
\lambda_{i}^{k}=\left\{\begin{array}{ll}
-g_{i}^{\prime}\left(\check{x}_{i}-\xi_{i}^{k}-\check{y}_{i}^{k}\right)+\check{\mu}_{i}^{k}, & \text { if } \check{x}_{i} \geq \xi_{i}^{k}+\check{y}_{i}^{k}, \\
f_{i}^{\prime}\left(\check{x}_{i}\right), & \text { otherwise, }
\end{array} \quad i \in I, k \in K\right.
$$


and $\check{\mu}_{i}^{k}$ is computed as follows:

$$
\check{\mu}_{i}^{k}= \begin{cases}\frac{\partial u_{i}}{\partial y_{i}}\left(\check{x}_{i}, \xi_{i}^{k}, \check{y}_{i}^{k}\right)-\check{\mu}_{n e}^{k}, & \text { if } \check{y}_{i}^{k}=\check{x}_{i}-\xi_{i}^{k}, \quad i \in I, k \in K \\ 0, & \text { otherwise, }\end{cases}
$$

and

$$
\check{\mu}_{n e}^{k}=\left\{\begin{array}{ll}
\frac{\partial u_{j}}{\partial y_{j}}\left(\check{x}_{i}, \xi_{i}^{k}, \check{y}_{i}^{k}\right), & \text { if } \exists j \text { such that } 0<\check{y}_{j}^{k}<\check{x}_{j}-\xi_{j}^{k}, \quad k \in K . \\
0, & \text { otherwise. }
\end{array} \quad k\right.
$$

In short, we apply Lagrangian duality to derive formula (10), and then apply KKT conditions on the $k^{\text {th }}$ recourse subproblem to calculate the optimal dual multipliers provided in (11)-(12). What makes our result particularly interesting is the fact that Theorem 1 allows computing the values of $\boldsymbol{\lambda}^{k}$ only using the optimal solutions of the subproblems, $\check{\boldsymbol{y}}^{k}$. Using the concavity of $U(\boldsymbol{x}, \boldsymbol{\xi}, \boldsymbol{y})$ in $\boldsymbol{y}$ for any given $\boldsymbol{x}$ and $\boldsymbol{\xi}$ (see Part 1 of Lemma 3 in the supplementary appendix), the subproblem of Model (2) can be solved efficiently using the current literature on concave allocation problems. In this way, we significantly decrease the computational effort by avoiding the use of generic nonlinear solvers to obtain the values of $\check{\boldsymbol{y}}$ and the associated dual multipliers. Our results in Section 5 provide computational evidence for this claim.

Initial upper bounds for $\boldsymbol{\theta}$. To initialize the cutting plane procedure, it is crucial to start with strong upper bounds for $\theta^{k}, k \in K$. Let $U B_{\theta}^{k}$ denote this initial bound, which is added to the master problem before any separation of cutting planes. For $U B_{\theta}^{k}$, we consider the utility obtained given the perfect information under scenario $k$ by solving the following optimization model. Note that in case of perfect information, we would have $x_{i}=\xi_{i}+y_{i}$ and therefore, $g_{i}\left(x_{i}-\alpha_{i}\right)=0$.

$$
U B_{\theta}^{k}=\max _{\boldsymbol{y} \geq 0}\left\{\sum_{i=1}^{n} f_{i}\left(\xi_{i}^{k}+y_{i}^{k}\right): \sum_{i=1}^{n} y_{i}^{k}=\xi_{n e}^{k}\right\} .
$$

Algorithmic details. To accelerate the convergence of the algorithm, we generate several optimality cuts before starting the GBD method. To that purpose, we first compute $\overline{\boldsymbol{x}}^{k}=$ $\arg \max _{\boldsymbol{x} \geq \mathbf{0}} Q\left(\boldsymbol{x}, \boldsymbol{\xi}^{k}\right)$ for each $k$, as the optimal solution under $\boldsymbol{\xi}^{k}$ and generate Benders cuts that potentially cut off these points. 
Since a cut can be generated for any pair $\overline{\boldsymbol{x}}^{k}$ and $\boldsymbol{\xi}^{k^{\prime}}$, for each $k, k^{\prime} \in K$, a total of $|K|^{2}$ initial cuts can be generated at this stage. However, to avoid overloading of the master problem with a large number of cuts, for each $\overline{\boldsymbol{x}}^{k}$, we randomly select $n$ possible realizations of $\boldsymbol{\xi}$ and solve the associated subproblem to obtain $n \cdot|K|$ initial optimality cuts. Given these cuts, we start the Benders decomposition algorithm and continue until we reach $\epsilon$ convergence, i.e., until we obtain a solution $(\check{\boldsymbol{x}}, \check{\boldsymbol{\theta}})$ such that $\check{\theta}^{k}-Q\left(\check{\boldsymbol{x}}, \check{\boldsymbol{\xi}^{k}}\right) \leq \epsilon$ holds, for all $k \in K$.

\subsection{Developing a fast heuristic}

Although performing the generalized Benders decomposition under a well-designed SAA scheme provides an optimal (or near-optimal) solution for Model (1), it may require considerable computational effort, especially for larger values of $n$ and $|K|$. Therefore, it is necessary to have an efficient heuristic that can find high-quality solutions in a short amount of time.

To that purpose, we decompose the budget target for delegation $i, x_{i}$, into two parts: $x_{i}^{\prime}$, which refers to the budget target solely from the earmarked donations for delegation $i$; and $x_{i}^{\prime \prime}$, which is an additional budget target from taking the nonearmarked donations into account. If all elements of $\boldsymbol{\xi}$ are independent (i.e., there exists no correlation in the donors' decision on earmarking for a certain delegation or not earmarking at all), then according to Proposition 3 , we can find $x_{i}^{\prime}$ by solving the following equation for each $i$ :

$$
\mathbb{B}_{i}\left(x_{i}^{\prime}\right)=\frac{f_{i}^{\prime}\left(x_{i}^{\prime}\right)}{f_{i}^{\prime}\left(x_{i}^{\prime}\right)+a_{i}^{g}}
$$

To find $\boldsymbol{x}^{\prime \prime}$, we maximize the overall beneficiaries' welfare, $\sum_{i} f_{i}\left(\alpha_{i}\right)$, by optimally allocating the expected value of nonearmarked donations, $\mathbb{E}\left(\tilde{\xi}_{n e}\right)$, among $n$ delegations, considering a previously allocated vector of $\boldsymbol{x}^{\prime}$ :

$$
\boldsymbol{x}^{\prime \prime}=\arg \max _{\boldsymbol{x}^{\prime \prime} \geq 0} \sum_{i=1}^{n} f_{i}\left(x_{i}^{\prime \prime}+x_{i}^{\prime}\right), \quad \text { s.t. } \quad \sum_{i=1}^{n} x_{i}^{\prime \prime}=\mathbb{E}\left(\tilde{\xi}_{n e}\right) .
$$

The above function is concave in $\boldsymbol{x}^{\prime \prime}$ and therefore can be solved easily. Given Equations (14) and (15), one can compute the heuristic solution as $\boldsymbol{x}^{h}=\boldsymbol{x}^{\prime}+\boldsymbol{x}^{\prime \prime}$.

\section{Numerical experiments}

In this section, we describe the data set and report on the results of the numerical computations. 


\subsection{Description of Data Set from ICRC}

To evaluate our model under a valid range of parameters, we use ICRC's annual reports to extract required data. These are comprehensive reports from the activities of ICRC on different delegations and are publicly available from its website. By the beginning of year 2018, ICRC operated on 57 main delegations (we skipped the New York delegation since it is ICRC's delegation to the UN). We focus on the data of the last six years, from 2012 to 2017, and interpolate missing values.

We used the same ratio of earmarked donations to total expenditure for estimating the donation distributions of the delegations; this implies that for the year 2012, $29 \%$ of total expenditure was from nonearmarked donations, while the rest is financed from earmarked donations. This ratio is $28 \%, 27 \%, 26 \%, 23 \%$ and $22 \%$ for years 2013 to 2017 . We consequently compute the mean and standard deviation for all earmarked and nonearmarked donations. We assume the mean nonearmarked budget to be $22 \%$ of the total expected donations (the ratio of 2017). The standard deviation is then easily computed using the coefficient of variation, which we already found for the nonearmarked donations. As in Okten and Weisbrod (2000), we assume that all donations, whether earmarked or not, follow log-normal distributions. All budget values are given in million Swiss franc (MCHF).

We assume that $f_{i}()=.a_{i}^{f}(.)^{b_{i}^{f}}$. To compute $a_{i}^{f}$, we assume that a delegation with more critical missions accomplished in the last year would be of higher priority for the ICRC in the current year. Therefore, we consider key performance metrics on 2017 that are reported by the ICRC for each delegation. After rescaling the values of each metric, an expert from a Canadian foundation assigned weight to each metric. The weighted sum of these values provides $a_{i}^{f}$ for each country.

To compute $b_{i}^{f}$, we look at the expenditure break down for each delegation in 2017 in five main categories, protection, assistance, prevention, cooperation with national societies and general. We assume that the more evenly the expenditures are divided among these areas, the more diverse are the operations on that delegation, and therefore, $b_{i}^{f}$ should be smaller. Thus, we compute $\frac{\text { maximum of the } 5 \text { expenditures }}{\text { average of the } 5 \text { expenditures }}$ for each country, followed by rescaling them between 0 and 1 by setting the largest value to 0.95 . This will guarantee that all welfare functions are concave. Alternatively, factor $b_{i}^{f}$ of each delegation $i$ can be computed using the Herfindahl-Hirschman Index (HHI) (Rhoades 1993) on the five main expenditures mentioned above and by rescaling the values. 
Finally, for $a_{i}^{g}$, we consider the logarithm of the total population who live in the domain of the delegation (using data from World Population Prospects 2017), as an indicator of awareness to penalize the unsatisfied targets. We then rescale $\boldsymbol{a}^{g}$ such that $\max _{i} a_{i}^{g}=\max _{i} a_{i}^{f}$ to make a balance between the two factors.

The parameters for all 57 delegations are presented in Appendix A. For problems with $n<57$ we use the first $n$ delegations with the highest targeted budget for 2018. In our data, the first 30 delegations represent approx. 85-90\% of the total spending; since $n=30$ is a better representative of an average IHO compared to $n=57$, we use it for the majority of our numerical experiments.

\subsection{Computational Performance}

To conduct our computations, we use MATLAB R2019a as well as Gurobi 9.0.2. We run the computations in a system that consists of a $2.7 \mathrm{GHz}$ Intel Core i7 processor with $16 \mathrm{~GB}$ of RAM and a MacOS Catalina.

Computational efficiency and sample stability: To evaluate the computational efficiency of the proposed new generalized Benders decomposition method presented in Section 4.2 and our analytical findings in Theorem 1, we compare it with a GBD algorithm in which an off-the-shelf nonlinear solver (MATLAB's fmincon) is used to solve the subproblems and calculate dual variables. We refer to the latter as $G B D-g$. We also report the computational performance of the extensive form method (cf. Section 4.1) in Appendix E of the supplementary document; the latter scales very poorly with the increasing size of $n$ and $|K|$, and is up to three orders of magnitude slower than GBD and up to two orders of magnitude slower than GBD-g.

Our results in Figure 1 indicate that for any $n$ and $|K|$, the CPU time for GBD is several times less than that of GBD-g. Therefore, using GBD (instead of GBD-g) allows for significant savings in computing time.

To evaluate the underlying scenario generating scheme and estimate the required sample size for achieving good and stable solutions, we use two related quality measures, namely, in-sample stability and out-of-sample stability (see, e.g., King and Wallace (2012), Ljubić et al. (2017)). We refer to the supplementary appendix for more details. We also show the adequacy of samples of size $|K|=500$ for our computation, and thus, we use $|K|=500$ for the following computations. 


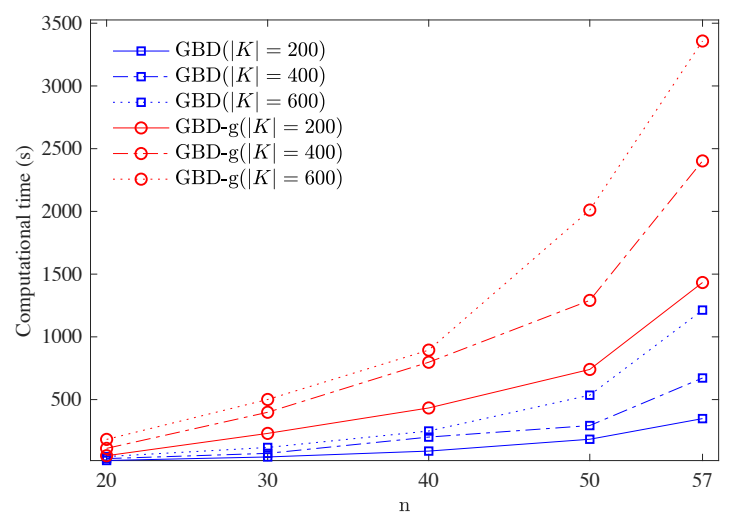

Figure 1 Comparison between GBD and GBD-g in terms of required CPU time.

Value of stochastic solution and other performance measures: We aim to answer the following questions: (1) why the IHO should not simplify the budgeting problem by assuming a deterministic (rather than a stochastic) model, (2) why the IHO should not simplify the budgeting problem by assuming a linear (rather than a non-linear concave) objective function?, and (3) what is the quality of solutions obtained by the heuristic proposed in Subsection 4.3?

To address these questions, we compare the IHO's utility under different budgeting plans, where each plan is obtained using a different modeling assumption. While the GBD method provides IHO's utility under our default setting (stochastic with non-linear objective), to achieve the maximum precision we replicate several iterations of GBD under Sample Average Approximation (SAA) framework. Please refer to Appendix $\mathrm{F}$ in the online supplementary material for more information about our SAA approach. $\left|K^{\prime}\right|=4000$ scenarios were used as the reference set in the SAA approach. For a fair comparison, we also used the same $K^{\prime}$ scenarios to evaluate the utilities of the solutions obtained with other methods. The next proposition (whose results can be derived from, e.g., Mangasarian and Rosen (1964)) helps to explore the first question.

Proposition 4. Let $\overline{\boldsymbol{\xi}}=\mathbb{E}(\boldsymbol{\xi})$ and $\overline{\boldsymbol{x}}$ be the optimal solution for $\max _{\boldsymbol{x} \geq \mathbf{0}} Q(\boldsymbol{x}, \overline{\boldsymbol{\xi}})$. We have the following:

$$
\underbrace{\mathbb{E}_{\tilde{\boldsymbol{\xi}}}[Q(\overline{\boldsymbol{x}}, \boldsymbol{\xi})]}_{E E V} \leq \mathfrak{Q}\left(\boldsymbol{x}^{*}\right) \leq \underbrace{\mathbb{E}_{\tilde{\boldsymbol{\xi}}}\left[\max _{\boldsymbol{x} \geq \mathbf{0}} Q(\boldsymbol{x}, \boldsymbol{\xi})\right]}_{W S} \leq Q(\overline{\boldsymbol{x}}, \overline{\boldsymbol{\xi}}) .
$$

The value $\overline{\boldsymbol{x}}$ in Proposition 4 is usually referred to as the Expected Value (EV) solution. The first term in Equation (16) identifies the Expected value of the EV solution (EEV). This is the maximum 
Table 1 The expected utility obtained by GBD embedded in the SAA framework, its lower and upper bounds (EV and WS respectively), the heuristic solution, and the expected value for a linear utility function.

\begin{tabular}{llllllllllll}
\hline & \multicolumn{3}{c}{ EV } & & GBD & \multicolumn{2}{c}{ WS } & \multicolumn{2}{c}{ Heuristic } & \multicolumn{2}{c}{ Linear UF } \\
\cline { 2 - 11 } $\mathbf{n}$ & utility & VSS & gap & utility & utility & EVPI [\%] & utility & gap & utility & gap \\
\hline 10 & 14079 & 1745 & $12.4 \%$ & 15824 & 18040 & $12.3 \%$ & 15431 & $2.5 \%$ & 14077 & $12.4 \%$ \\
20 & 15772 & 2576 & $16.3 \%$ & 18347 & 20902 & $12.2 \%$ & 17910 & $2.4 \%$ & 15681 & $17.0 \%$ \\
30 & 16080 & 3200 & $19.9 \%$ & 19280 & 21944 & $12.1 \%$ & 18825 & $2.4 \%$ & 16295 & $18.3 \%$ \\
40 & 16377 & 3433 & $21.0 \%$ & 19810 & 22574 & $12.2 \%$ & 19397 & $2.1 \%$ & 16719 & $18.5 \%$ \\
50 & 16556 & 3592 & $21.7 \%$ & 20148 & 22916 & $12.1 \%$ & 19703 & $2.3 \%$ & 16896 & $19.2 \%$ \\
57 & 16768 & 3567 & $21.3 \%$ & 20335 & 23148 & $12.2 \%$ & 19891 & $2.2 \%$ & 16967 & $19.9 \%$ \\
\hline
\end{tabular}

utility that can be achieved by solving a deterministic instead of a stochastic problem, in which the uncertain donations are replaced by their expected values. The larger the gap between EEV and $\mathfrak{Q}\left(\boldsymbol{x}^{*}\right)$, the more important it is to solve the stochastic problem. The value of the stochastic solution (VSS) is defined as $V S S=\mathfrak{Q}\left(\boldsymbol{x}^{*}\right)-E E V$, and is reported in Table 1 in its absolute and relative terms. We observe that there is a significant benefit from considering donation uncertainty in our model: the stochastic solution outperforms the EV solution by a large margin (i.e., the IHO could loose up to $21.7 \%$ of its utility, on average, if the decision maker adopts a simplified modeling by replacing the uncertain donations by their expected values).

If it was possible to predict in advance all uncertainties, one would achieve the maximum possible utility, which provides a theoretical upper bound to $\mathfrak{Q}\left(\boldsymbol{x}^{*}\right)$. Such obtained Wait and See (WS) solution value corresponds to the third term in Equation $(16), W S=\mathbb{E}_{\tilde{\boldsymbol{\xi}}}\left[\max _{\boldsymbol{x} \geq \mathbf{0}} Q(\boldsymbol{x}, \boldsymbol{\xi})\right]$. In Table 1 we report the utility of the WS solution, and we also compute the Expected Value of Perfect Information (EVPI) [\%], which is the relative loss of utility with respect to the value of WS due to the lack of access to the perfect information, i.e., $E V P I[\%]=\left(W S-\mathfrak{Q}\left(\boldsymbol{x}^{*}\right)\right) / W S * 100$. As we can observe from Table 1, the IHO looses around $12 \%$ of its utility, on average, due to the inability to perfectly predict the donations.

To answer the second question regarding the impact of simplifying the concave utility function with a linear function, we calculate the optimal solution, $\boldsymbol{x}^{L}$, for $b_{i}^{f}=1$ for all $i$, and its expected utility $\mathbb{E}_{\tilde{\boldsymbol{\xi}}}\left[Q\left(\boldsymbol{x}^{L}, \boldsymbol{\xi}\right)\right]$. The latter value is reported in Table 1 in the column denoted by Linear UF. Our results demonstrate that considering linear utility functions for the delegations results in significantly sub-optimal outcomes, with potential utility losses ranging between $12.4 \%$ and $19.9 \%$ in our experiments, which are nearly as high as the losses incurred by the EV solution. We conclude 
that it is important for the IHO to use a modeling approach that takes the concavity of the utility function into account.

Finally, to answer the third question, Table 1 also reports the expected utility of the heuristic solution, together with the relative gap between this value and the value of $\mathfrak{Q}\left(\boldsymbol{x}^{*}\right)$. The obtained results indicate that our heuristic approach provides solutions with small optimality gaps (ranging between $2.1 \%$ and $2.5 \%)$ in a short time $(0.22$ seconds for $n=57)$. The required computational time for GBD is about 16 minutes, and for GBD under the SAA framework is nearly 3 hours (with $n=57$ and $|K|=500)$. These results indicate that the heuristic can be successfully used for IHOs with a large number of delegations without a significant loss of the expected utility, when the exact methods cannot handle the problem.

From Table 1, we can also see that the overall utility of the IHO does not increase significantly by expanding the operations into more countries (with less urgent missions). For example, by accomplishing the operations in the first 10 countries of the list in Table A.1, ICRC can achieve $78 \%$ of the utility that it can obtain by conducting operations in all of its delegations. Note that while these 10 countries only represent $7.1 \%$ of the world's population, they are engaged in the worst humanitarian crises. Considering the costs of operating in new countries, this finding may explain why the majority of the IHOs focus on a small number of countries.

\subsection{Sensitivity Analyses and Managerial Insights}

In this subsection we present two sensitivity analyses concerning the impact of the degree of earmarking and the impact of correlation among donations. For additional analyses of the impact of the parameters of the utility functions and the impact of budget uncertainty please see the supplementary appendix. The GBD method has been used to derive these sensitivity analyses. Note that our numerical findings are specific to the data set that we used.

Impact of the degree of earmarking: To study the performance of the IHO under different shares of the earmarked budget, we vary $\frac{\mathbb{E}\left(\xi_{n e}\right)}{\mathbb{E}\left(\xi_{n e}\right)+\sum_{i} \mathbb{E}\left(\xi_{i}\right)}$ from $5 \%$ to $80 \%$.

In each scenario, we keep $\sum_{i} \mathbb{E}\left(\xi_{i}\right)+\mathbb{E}\left(\xi_{n e}\right)$ the same. We then proportionally change the means and standard deviations of all elements of $\boldsymbol{\xi}$.

Figure 2 demonstrates the importance of nonearmarked donations on the performance of the IHOs under different values of $n$. Figure 2 a shows that by convincing more donors not to earmark 


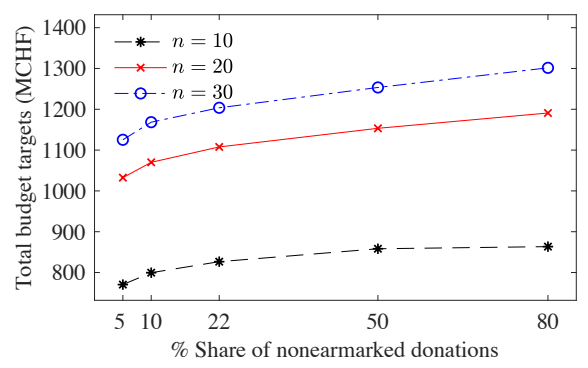

(a) Sum of budget targets

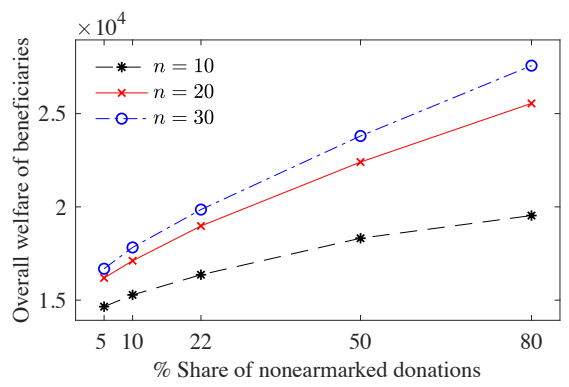

(b) Overall welfare of beneficiaries

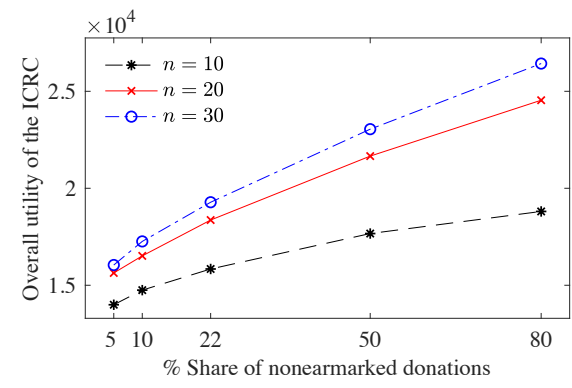

(c) Overall utility of the IHO

Figure 2 The impact of the share of the nonearmarked donations on (a) budget targets, (b) beneficiaries' welfare, and (c) IHO's utility.

their donations, the IHO would target significantly larger plans, since the managers are now less worried about fulfilling the targets. The improvement is especially sharp under smaller shares of the nonearmarked budget. Higher budget targets would in turn lead to higher welfare of the beneficiaries (Figure 2b). The latter, combined with a higher fulfillment of initial plans (due to having more flexibility in budget allocation), results in a higher overall utility for the IHO (Figure 2c). We can also see that larger IHOs benefit more from a higher share of nonearmarked funding, which is in line with our findings of Proposition 1 .

We can observe a $15.7 \%$ increase in the total budget targets as well as a $65.3 \%$ improvement in the overall welfare of beneficiaries when moving from a $5 \%$ share of the nonearmarked budget to $80 \%$ (for $n=30$ ), indicating the benefit of nonearmarked donations.

Impact of correlation among donations: In some settings, donors for the $n$ delegations and the nonearmarked donations may be overlapping, and the delegations may be in competition for donations, depending on the ongoing crises in the different countries. Some donors may also hesitate between donating earmarked funds and nonearmarked funds to the IHO. These settings lead to correlation in the donations.

We test the effect of correlation in a numerical experiment, assuming correlation between the delegations' earmarked donations, $\xi_{i}$, and the nonearmarked donations, $\xi_{n e}$, and with different earmarked donations being independent with respect to each other. For simplicity, we assume the same correlation coefficient $\rho$ for all $i$. The maximum possible correlation $\rho$ for $n+1$ distributions is $\pm|1 / \sqrt{n}|$.

The results of our experiments are shown in Figure 3 and indicate that the IHO benefits from negative correlation between earmarked and nonearmarked distributions, which is in line with 


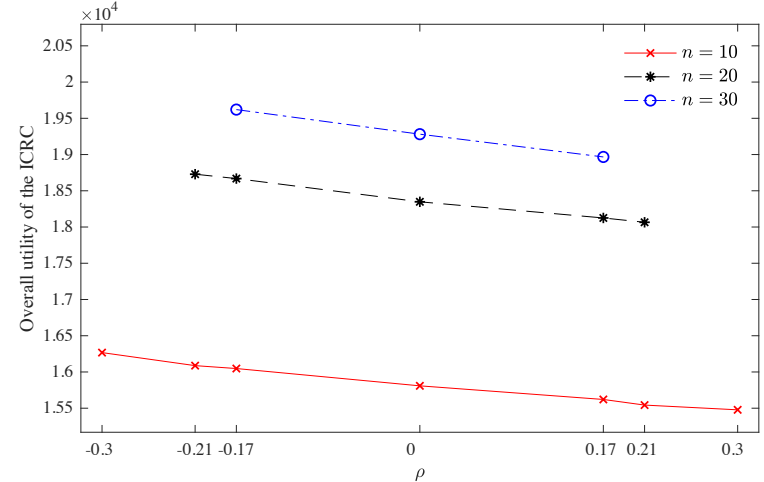

(a) Overall utility of the IHO

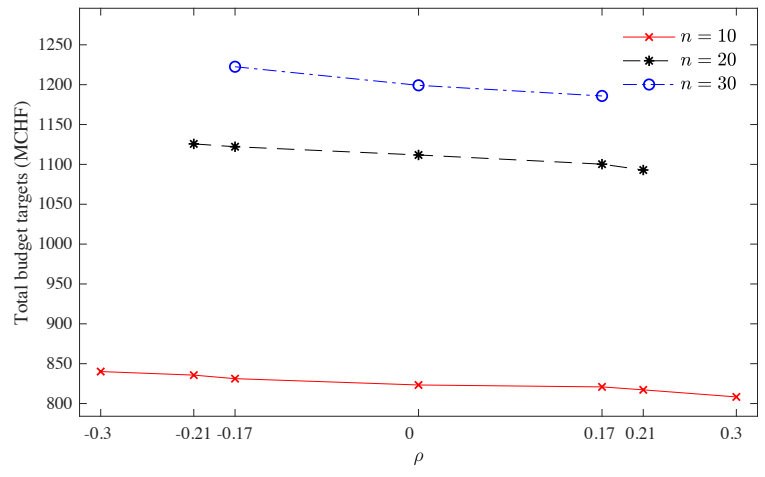

(b) Sum of budget targets

Figure 3 The impact of negative and positive correlation between earmarked and nonearmarked donations on (a) IHO's utility and (b) the expected sum of budget targets.

expectations and prior research on allocation problems; see, for example, Netessine et al. (2002). The effect is the opposite in case of positive correlations. The results also relate to the findings of Acharya et al. (2006) which emphasizes the necessity of coordination among donors to enhance the aid efficiency.

We also observe from Figure 3 that negative correlation leads to higher targets and vice versa. For example, for $n=30$ delegations, the sum of targets increases by $1.9 \%$ when moving from zero to the maximum negative correlation, while it decreases by $1.1 \%$ when moving to the maximum positive correlation. In fact, negative correlation implies that whenever delegation $i$ receives few earmarked donations, the IHO is more likely to receive more nonearmarked donations and vice versa. Consequently, the IHO is able to set a higher target and to fulfill the target either with earmarked or with nonearmarked donations depending on the distributional outcome. An opposite mechanism applies to settings with positive correlations. We also observed in our experiments that correlation among donations does not significantly affect the optimality gap of our heuristic solution.

\section{Extension to Target-Dependent Donations}

In this section, we extend our model to a setting where the distribution of earmarked donations for each delegation $i$ depends on the target for that delegation, $x_{i}$. This extension models situations in which the IHO's fundraising efforts are affected by the target it has set and in which a high target 
motivates donors to give more. The objective function of the extended model can be written as follows:

$$
\max _{\boldsymbol{x} \geq 0} \mathscr{Q}(\boldsymbol{x})=\mathbb{E}_{\tilde{\boldsymbol{\xi}} \mid \boldsymbol{x}}[Q(\boldsymbol{x}, \boldsymbol{\xi})]
$$

This extension can be considered a Stackelberg game, in which the IHO decides first about the budget targets, anticipating the reaction of the donors, and the donors decide in a second step on the donations as a function of the targets. In its generic form as presented in Equation (17), the distribution of donations depends on the decision variables, and thus the resulting problem belongs to the class of stochastic programming with decision-dependent uncertainty, which is fairly new in the stochastic programming literature (Hellemo et al. 2018). In this model, $\mathscr{Q}(\boldsymbol{x})$ is not concave in $\boldsymbol{x}$, and thus, we cannot use standard convex optimization techniques to derive the optimal $\boldsymbol{x}$. A full investigation of this generic model would require a new research paper. Consequently, in this paper, we propose a simple model and heuristic solution approach using the Nelder-Mead Simplex algorithm to address the new problem. Details of the model and solution approach can be found in Appendix C.

Next, we run numerical experiments to analyse the extended model. To that purpose, we assume a linear relationship between the mean of the earmarked donations and the budget target for a given delegation $i, \mathbb{E}\left(\xi_{i}\right)=d \bar{w}_{i}+e x_{i}$, where $\bar{w}_{i}$ is the default mean of earmarked donations that was extracted from ICRC data (cf. Table A.1), $d \bar{w}_{i}$ is the line's intercept, and $e$ represents the slope. The slope can be interpreted as the donors' sensitivity to budget targets, while the intercept represents the minimum expected budget that the IHO can expect even with a zero target. For simplicity, we assume common parameters $d$ and $e$ for all delegations. The coefficient of variation of each distribution remains the same as the one implied by Table A.1, and similar to Section 5 . we assume a Lognormal distribution for all donations. We also assume that $\xi_{n e}$ is independent of $\boldsymbol{x}$. We use the heuristic approach presented in Appendix $\mathbb{C}$ for the computations.

Furthermore, we analyze the impact of partially and fully relaxed earmarking schemes that we introduced in Section 3.2. The partially relaxed earmarking scheme is especially relevant under the assumption of target-dependent donations, because it affects the Stackelberg game between the IHO and the donors. Note that Corollary 1 is still valid under the assumption of target dependent donations, i.e., $\mathscr{Q}(\boldsymbol{x}) \leq \max _{\boldsymbol{x} \geq \mathbf{0}} \mathbb{E}_{\tilde{\boldsymbol{\xi}} \mid \tilde{\boldsymbol{x}}}\left[Q^{\prime}(\boldsymbol{x}, \boldsymbol{\xi})\right] \leq \max _{\boldsymbol{x} \geq \mathbf{0}} \mathbb{E}_{\tilde{\boldsymbol{\xi}} \mid \tilde{\boldsymbol{x}}}\left[Q^{\prime \prime}(\boldsymbol{x}, \boldsymbol{\xi})\right]$. 


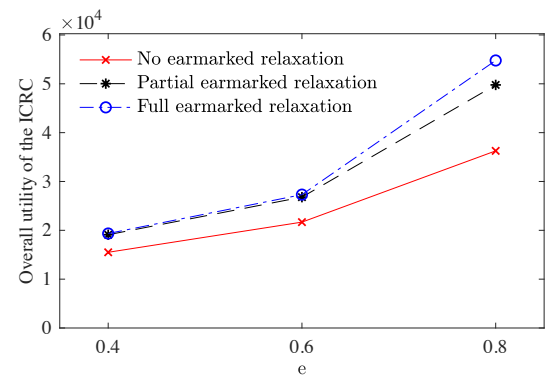

(a) $d=0.25$

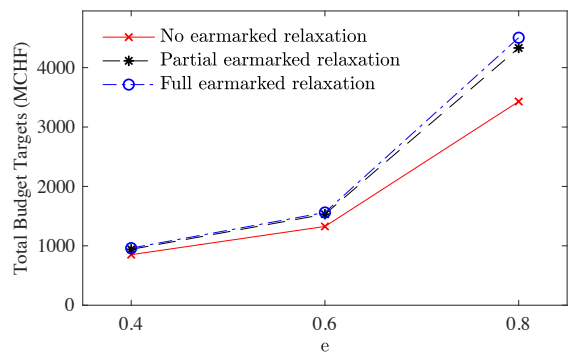

(c) $d=0.25$

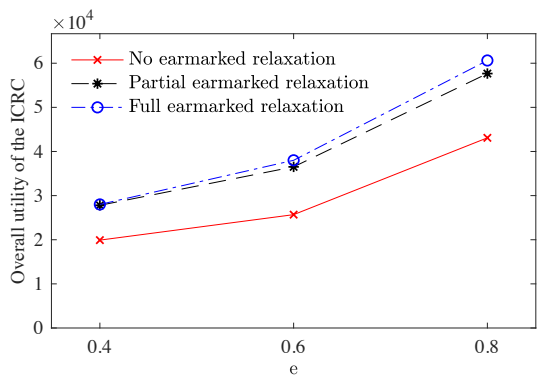

(b) $d=0.5$

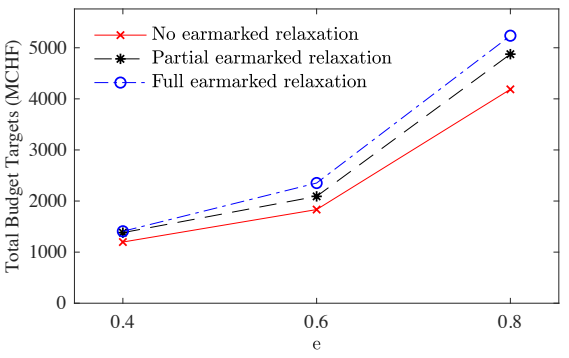

(d) $d=0.5$

Figure $4 \quad$ IHO utility and total budget targets as a function of slope $e$ for different values of intercept $d$ and for different relaxation schemes (none, partial, full) with $n=30$.

Figure 4 demonstrates the results. Concerning the relationship between the distribution of donations and budget targets, our results indicate that as the slope $e$, i.e., the donor sensitivity to call for donations, increases, the IHO sets higher targets at a more aggressive rate (Figure 4 c and $4 \mathrm{~d}$ ). This implies a faster increase in the IHO's utility (see Figures $4 \mathrm{a}$ and $4 \mathrm{~b}$ ). Our insights highlight the importance of measures to increase the slope $e$, such as with the IHO's fundraising efforts, the visibility of the IHO and its missions to potential donors, and by obtaining donors' trust in the IHO's credibility and capabilities. On the other hand, comparing Figure $4 \mathrm{a}$ with Figure $4 \mathrm{~b}$, shows that increasing the intercept multiplier $d$ may not be as beneficial as increasing slope $e$ for the IHOs. In other words, investing on factors that impact the awareness of donors about IHO's missions could be more beneficial than securing donations from additional sources.

Figures $4 \mathrm{a}$ and $4 \mathrm{~b}$ also indicate that by using the partial earmarking scheme, the IHO can obtain a utility close to the scheme with no earmarking constraints, performing significantly $(33 \%$ in average over the test problems) better than under the original scheme with full earmarking constraints. This effect increases with increasing slope $e$, i.e., it becomes more important with stronger dependency of donations on the targets, and also with increasing intercept $d$. The partially 
relaxed earmarking scheme also leads the IHO to set targets that are significantly higher than those under the original scheme (see Figures 4 c and $4 \mathrm{~d}$ ). This important finding implies that IHOs should ask (and incentivize) donors to give permission to reallocate donations after targets are fulfilled.

Indeed, many IHOs such as ICRC ask donors at the end of the year to allow for the reallocation or return of earmarked donations that have not been spent. What we propose is to ask the permission at the beginning of the year, which gives the IHO more flexibility in setting targets and spending the budget. Donations under this scheme have an efficiency close to that of non-earmarked donations, and compared to earmarked donations, our scheme could be more attractive for donors, because the IHO commits to fully meeting the targets of the specified use.

\section{Discussion and Conclusion}

In this research, we study budgeting in international humanitarian organizations, which is primordial to improve beneficiaries' welfare. We model the problem as a nonlinear two-stage stochastic program with recourse and develop an efficient generalized Benders decomposition algorithm for the problem that outperforms other alternatives in terms of computational efficiency. Our results indicate a significant improvement over the expected value solution $(21.3 \%$ optimality gap for the largest problem in our tests), which highlights the importance of taking the stochastic nature of the problem into account. We also propose a heuristic approach that can find close-to-optimal solutions (2.2\% optimality gap when considering all delegations), and can be easily adapted to IHOs that run numerous delegations. We also developed two model extensions to consider budget adjustments during the year and budgeting under target-dependent donations.

We derived a number of interesting managerial insights, both analytically and numerically. Our study confirms existing research that nonearmarked donations have a higher contribution on beneficiaries' welfare and IHOs' performance, compared to the earmarked donations. The current trend that donors earmark their donations forces the IHOs to set lower targets, resulting in beneficiaries being worse off, ceteris paribus. With the analysis of our data set, we find for example that if the share of nonearmarked donations decreases from $22 \%$ to $5 \%$, ICRC should set its budget targets $6.5 \%$ lower for the main 30 delegations (leading to a decrease of $16.0 \%$ of welfare). 
We observed that large IHOs gain more from nonearmarked donations than smaller IHOs, for which earmarked donations create more inefficiency. For example, an IHO with 30 delegations, compared to one with 10 delegations, will experience a $7.9 \%$ greater increase in beneficiaries' welfare ( $19.9 \%$ vs $12 \%$ ) from moving from $22 \%$ share of nonearmarked donations to a $50 \%$ share.

We showed that the more the donors are concerned about the fulfillment of targets for a given delegation or the more diverse the operations in a delegation are, the lower the target that IHO sets for that delegation, which in turn leads to less welfare for the beneficiaries. In our experiments, a $50 \%$ increase of the penalty factor on unmet targets leads to a $3.3 \%$ reduction of budget targets for the main 30 delegations. Therefore, interestingly, it is better for the donors not to be strict on getting the IHO responsive for the targets it sets.

We also proposed a donation scheme that can significantly improve IHO performance, by $33 \%$ in our numerical experiments. Under this scheme, donations earmarked to a delegation can be allocated to other delegations if the declared budget target for the delegation is met. Note that the IHO can still benefit from this scheme if only a fraction of the donors of earmarking donations adopt it. Knowing the distribution of flexible and nonflexible earmarked donations, our model can be applied with few modifications to calculate the optimal targets.

We extended our model to the possibility to update budget targets during the year. We found that this extension leads to, on average, higher targets than under the standard model. In addition, an IHO may face unpredictable events during the year for some delegations, e.g., in case of disasters or disease outbreaks. In such a case, when the situation arises, the IHO can use our model and reoptimize the budget targets based on updated utility functions and donation distributions and also taking into account the donations already received by the time of event. The solutions methods that we proposed in this paper can be adapted to derive optimal solutions for the updated model which are then implemented from the time of the event.

Our research can be further developed in a number of directions. First, empirical and analytical studies are required to better understand the beneficiaries' welfare function in the context of humanitarian programs. Second, the concave utility function can be replaced with an S-shaped scheme, given the marginal welfare resulting from initial investments. Third, the problem can be extended to consider a dynamic setting with multiple time periods in which the distribution of donations in future periods is a function of the IHO's performance in previous years. 


\section{Acknowledgement}

We would like to thank Linda Romano from Development and Peace- Caritas Canada for her contribution in the estimation of model parameters. We are also grateful to Prof. Luk Van Wassenhove, as well as the anonymous associate editor and two referees whose many suggestions helped improve this study significantly.

\section{References}

Acharya, A., De Lima, A. T. F., and Moore, M. (2006). Proliferation and fragmentation: Transactions costs and the value of aid. The journal of development studies, 42(1):1-21.

Aflaki, A. and Pedraza-Martinez, A. J. (2016). Humanitarian funding in a multi-donor market with donation uncertainty. Production and Operations Management, 25(7):1274-1291.

Anheier, H. K. and Ben-Ner, A. (2003). The study of nonprofit enterprise: Theories and approaches. Springer Science \& Business Media.

Bansal, S. and Dyer, J. S. (2017). Multivariate partial-expectation results for exact solutions of two-stage problems. Operations Research, 65(6):1526-1534.

Benders, J. F. (1962). Partitioning procedures for solving mixed-variables programming problems. Numerische Mathematik, 4(1):238-252.

Berenguer, G., Iyer, A. V., and Yadav, P. (2016). Disentangling the efficiency drivers in country-level global health programs: An empirical study. Journal of Operations Management, 45:30-43.

Berenguer, G. and Shen, Z.-J. (2019). Challenges and strategies in managing nonprofit operations: An operations management perspective. Manufacturing 8 Service Operations Management, 22(5):8691106.

Besiou, M., Pedraza-Martinez, A. J., and Van Wassenhove, L. N. (2014). Vehicle supply chains in humanitarian operations: Decentralization, operational mix, and earmarked funding. Production and Operations Management, 23(11):1950-1965.

Besiou, M. and Van Wassenhove, L. N. (2020). Humanitarian operations: A world of opportunity for relevant and impactful research. Manufacturing \& Service Operations Management, 22(1):135-145.

Bhattacharya, S., Hasija, S., and Van Wassenhove, L. N. (2014). Designing efficient infrastructural investment and asset transfer mechanisms in humanitarian supply chains. Production and Operations Management, 23(9):1511-1521. 
Birge, J. R. and Louveaux, F. (2011). Introduction to stochastic programming. Springer Science \& Business Media.

Buse, K. and Walt, G. (1996). Aid coordination for health sector reform: a conceptual framework for analysis and assessment. Health Policy, 38(3):173-187.

Carøe, C. C. and Tind, J. (1998). L-shaped decomposition of two-stage stochastic programs with integer recourse. Mathematical Programming, 83:451-464.

Collier, P. and Dollar, D. (2002). Aid allocation and poverty reduction. European economic review, 46(8):1475-1500.

De Vericourt, F. and Lobo, M. S. (2009). Resource and revenue management in nonprofit operations. Operations research, 57(5):1114-1128.

Devalkar, S. K., Sohoni, M. G., and Arora, P. (2017). Ex-post funding: How should a resource-constrained non-profit organization allocate its funds? Production and Operations Management, 26(6):1035-1055.

Eichenauer, V. Z. and Reinsberg, B. (2017). What determines earmarked funding to international development organizations? Evidence from the new multi-bi aid data. Review of International Organizations, $12(2): 171-197$.

Erlebacher, S. J. (2000). Optimal and heuristic solutions for the multi-item newsvendor problem with a single capacity constraint. Production and Operations Management, 9(3):303-318.

Fischetti, M., Ljubić, I., and Sinnl, M. (2016). Benders decomposition without separability: A computational study for capacitated facility location problems. European Journal of Op. Research, 253(3):557-569.

Fischetti, M., Ljubić, I., and Sinnl, M. (2017). Redesigning Benders decomposition for large-scale facility location. Management Science, 63(7):2146-2162.

Halman, N., Orlin, J. B., and Simchi-Levi, D. (2012). Approximating the nonlinear newsvendor and singleitem stochastic lot-sizing problems when data is given by an oracle. Operations Research, 60(2):429-446.

Hellemo, L., Barton, P. I., and Tomasgard, A. (2018). Decision-dependent probabilities in stochastic programs with recourse. Computational Management Science, 15(3-4):369-395. 
Holguín-Veras, J., Pérez, N., Jaller, M., Van Wassenhove, L. N., and Aros-Vera, F. (2013). On the appropriate objective function for post-disaster humanitarian logistics models. Journal of Operations Management, $31(5): 262-280$.

Hyndman, N. and McDonnell, P. (2009). Governance and charities: An exploration of key themes and the development of a research agenda. Financial Accountability 83 Management, 25(1):5-31.

Jahre, M. and Heigh, I. (2008). Does the current constraints in funding promote failure in humanitarian supply chains? In Supply Chain Forum: An Intnl Journal, volume 9, pages 44-54. Taylor \& Francis.

Keshvari Fard, M., Eftekhar, M., and Papier, F. (2019). A policy for managing operational assets to minimize deprivation costs. Production and Operations Management, 28(8):2132-2151.

King, A. J. and Wallace, S. W. (2012). Modeling with Stochastic Programming. Springer.

Leitner, M., Ljubić, I., Luipersbeck, M., and Sinnl, M. (2018). Decomposition methods for the two-stage stochastic Steiner tree problem. Computational Optimization and Applications, 69(3):713-752.

Liu, C., Fan, Y., and Ordóñez, F. (2009). A two-stage stochastic programming model for transportation network protection. Computers \& Operations Research, 36(5):1582 - 1590.

Ljubić, I., Mutzel, P., and Zey, B. (2017). Stochastic survivable network design problems: Theory and practice. European Journal of Operational Research, 256(2):333-348.

Malik, K. (2018). As a system, foreign aid is a fraud and does nothing for inequality. The Guardian.

Mangasarian, O. and Rosen, J. (1964). Inequalities for stochastic nonlinear programming problems. Operations Research, 12(1):143-154.

McCardle, K. F., Rajaram, K., and Tang, C. S. (2009). A decision analysis tool for evaluating fundraising tiers. Decision Analysis, 6(1):4-13.

Natarajan, K. V. and Swaminathan, J. M. (2014). Inventory Management in Humanitarian Operations: Impact of Amount, Schedule, and Uncertainty in Funding. Manufacturing $\mathcal{E}$ Service Operations Management, pages 595-603.

Netessine, S., Dobson, G., and Shumsky, R. A. (2002). Flexible service capacity: Optimal investment and the impact of demand correlation. Operations Research, 50(2):375-388. 
Okten, C. and Weisbrod, B. A. (2000). Determinants of donations in private nonprofit markets. Journal of Public Economics, 75(2):255-272.

Patriksson, M. (2008). A survey on the continuous nonlinear resource allocation problem. European Journal of Operational Research, 185(1):1-46.

Pedraza-Martinez, A. J. and Van Wassenhove, L. N. (2013). Vehicle Replacement in the International Committee of the Red Cross. Production and Operations Management, 22(2):365-376.

Petrovits, C. M. (2006). Corporate-sponsored foundations and earnings management. Journal of Accounting and Economics, 41(3):335-362.

Privett, N. and Erhun, F. (2011). Efficient funding: Auditing in the nonprofit sector. Manufacturing E Service Operations Management, 13(4):471-488.

Rhoades, S. A. (1993). The herfindahl-hirschman index. Fed. Res. Bull., 79:188.

Ribar, D. C. and Wilhelm, M. O. (2002). Altruistic and joy-of-giving motivations in charitable behavior. Journal of political Economy, 110(2):425-457.

Takriti, S. and Ahmed, S. (2004). On robust optimization of two-stage systems. Mathematical Programming, $99(1): 109-126$.

Tomasini, R. and Van Wassenhove, L. (2009). Humanitarian logistics. Springer.

Toyasaki, F. and Wakolbinger, T. (2014). Impacts of earmarked private donations for disaster fundraising. Annals of Operations Research, 221(1):427-447.

Van Wassenhove, L. N. (2006). Humanitarian aid logistics: supply chain management in high gear. Journal of the Operational research Society, 57(5):475-489.

Varian, H. R. (1994). Sequential contributions to public goods. Journal of Public Economics, 53(2):165-186.

Wakolbinger, T., Toyasaki, F., Christopher, M., and Tatham, P. (2011). Impacts of funding systems on humanitarian operations. In Humanitarian logistics: meeting the challenge of preparing for and responding to disasters, pages 33-46. Kogan Page Limited London.

You, F. and Grossmann, I. E. (2013). Multicut benders decomposition algorithm for process supply chain planning under uncertainty. Annals of Operations Research, 210(1):191-211.

Zipkin, P. H. (1980). Simple ranking methods for allocation of one resource. Mgmt Science, 26(1):34-43. 
Online appendix for Budgeting in International Humanitarian Organizations

Appendix A: Parameters used for computations

Table A.1 Parameters used for computations

\begin{tabular}{|c|c|c|c|c|c|c|}
\hline No & Delegation & $\begin{array}{l}\text { mean } \\
\text { earmarked } \\
(\mathrm{MCHF})\end{array}$ & $\begin{array}{l}\text { std. dev. } \\
\text { earmarked } \\
(\mathrm{MCHF})\end{array}$ & $a_{i}^{f}$ & $b_{i}^{f}$ & $a_{i}^{g}$ \\
\hline 1 & Syrian Arab Republic & 138.651 & 41.447 & 30.592 & 0.950 & 51.509 \\
\hline 2 & Iraq & 99.863 & 23.961 & 60.688 & 0.781 & 53.787 \\
\hline 3 & South Sudan & 90.580 & 32.590 & 20.497 & 0.815 & 50.359 \\
\hline 4 & Yemen & 60.760 & 16.679 & 60.794 & 0.809 & 52.852 \\
\hline 5 & Nigeria & 74.427 & 30.195 & 36.592 & 0.828 & 58.738 \\
\hline 6 & Somalia & 72.458 & 11.533 & 36.975 & 0.918 & 50.848 \\
\hline 7 & Congo, Democratic Republic of the & 52.099 & 3.353 & 31.787 & 0.679 & 56.110 \\
\hline 8 & Afghanistan & 58.580 & 4.168 & 65.066 & 0.742 & 53.558 \\
\hline 9 & Ukraine & 41.389 & 20.574 & 13.826 & 0.790 & 54.232 \\
\hline 10 & Myanmar & 30.796 & 9.744 & 21.976 & 0.682 & 54.812 \\
\hline 11 & Israel and the Occupied Territories & 38.640 & 5.413 & 28.240 & 0.478 & 50.518 \\
\hline 12 & Central African Republic & 33.697 & 9.374 & 4.971 & 0.815 & 47.300 \\
\hline 13 & Mali & 31.270 & 4.648 & 14.881 & 0.792 & 51.555 \\
\hline 14 & Lebanon & 34.189 & 11.518 & 13.492 & 0.784 & 48.121 \\
\hline 15 & Libya & 26.176 & 6.373 & 12.667 & 0.774 & 48.266 \\
\hline 16 & Niger & 24.317 & 6.995 & 11.601 & 0.839 & 52.008 \\
\hline 17 & Jordan & 23.304 & 10.228 & 3.838 & 0.758 & 49.560 \\
\hline 18 & Colombia & 22.298 & 1.287 & 10.716 & 0.494 & 54.553 \\
\hline 19 & Yaoundé (regional) & 18.705 & 6.710 & 6.433 & 0.671 & 53.313 \\
\hline 20 & Mexico City (regional) & 17.365 & 3.916 & 7.132 & 0.501 & 58.869 \\
\hline 21 & Bangladesh & 10.892 & 3.368 & 12.650 & 0.693 & 58.282 \\
\hline 22 & Ethiopia & 14.681 & 2.340 & 17.784 & 0.577 & 56.895 \\
\hline 23 & Philippines & 13.527 & 11.718 & 10.506 & 0.536 & 56.894 \\
\hline 24 & Pakistan & 12.932 & 3.352 & 29.959 & 0.573 & 58.835 \\
\hline 25 & Abidjan (regional) & 8.086 & 1.280 & 6.573 & 0.375 & 56.465 \\
\hline 26 & Beijing (regional) & 10.677 & 1.897 & 3.972 & 0.507 & 65.066 \\
\hline 27 & Sudan & 6.869 & 8.980 & 10.448 & 0.337 & 53.964 \\
\hline 28 & Bangkok (regional) & 10.233 & 0.557 & 6.050 & 0.401 & 58.680 \\
\hline 29 & Pretoria (regional) & 6.452 & 2.002 & 0.971 & 0.509 & 57.360 \\
\hline 30 & Moscow (regional) & 11.840 & 3.798 & 0.300 & 0.471 & 58.146 \\
\hline 31 & New Delhi (regional) & 9.985 & 0.609 & 10.833 & 0.398 & 64.808 \\
\hline 32 & Chad & 8.594 & 3.274 & 3.387 & 0.549 & 50.881 \\
\hline 33 & Burundi & 7.772 & 2.222 & 10.716 & 0.543 & 49.908 \\
\hline 34 & Nairobi (regional) & 9.876 & 1.925 & 10.616 & 0.347 & 56.982 \\
\hline 35 & Paris (regional) & 8.169 & 2.252 & 2.397 & 0.583 & 59.543 \\
\hline 36 & Tashkent (regional) & 9.864 & 0.617 & 2.494 & 0.463 & 55.684 \\
\hline 37 & Suva (regional) & 6.937 & 1.239 & 2.094 & 0.329 & 53.916 \\
\hline 38 & Azerbaijan & 7.620 & 1.178 & 1.257 & 0.578 & 49.599 \\
\hline 39 & Dakar (regional) & 6.320 & 0.854 & 0.928 & 0.423 & 51.843 \\
\hline 40 & Brasilia (regional) & 7.709 & 1.048 & 1.223 & 0.312 & 59.938 \\
\hline 41 & Sri Lanka & 6.819 & 1.503 & 3.604 & 0.456 & 51.920 \\
\hline 42 & Washington (regional) & 5.561 & 0.412 & 0.033 & 0.588 & 60.701 \\
\hline 43 & Georgia & 5.747 & 0.777 & 5.643 & 0.534 & 46.762 \\
\hline 44 & Caracas (regional) & 3.336 & 0.767 & 0.038 & 0.425 & 53.866 \\
\hline 45 & Kuala Lumpur (regional) & 5.071 & 0.855 & 1.587 & 0.509 & 58.293 \\
\hline 46 & Kuwait (regional) & 5.042 & 1.121 & 1.077 & 0.386 & 54.767 \\
\hline 47 & Tunis (regional) & 4.430 & 0.568 & 2.645 & 0.462 & 50.236 \\
\hline 48 & Balkans (regional) & 4.475 & 0.780 & 1.664 & 0.722 & 54.670 \\
\hline 49 & Iran & 3.757 & 0.440 & 0.550 & 0.381 & 56.103 \\
\hline 50 & Rwanda & 4.225 & 0.365 & 11.176 & 0.493 & 50.267 \\
\hline 51 & Lima (regional) & 4.297 & 0.356 & 7.380 & 0.372 & 55.164 \\
\hline 52 & Uganda & 3.186 & 0.433 & 7.348 & 0.516 & 54.136 \\
\hline 53 & Egypt & 3.271 & 1.124 & 2.357 & 0.323 & 56.670 \\
\hline 54 & London & 3.069 & 0.689 & 0.306 & 0.437 & 55.688 \\
\hline 55 & Armenia & 2.588 & 0.470 & 0.265 & 0.408 & 45.872 \\
\hline 56 & Jakarta (regional) & 3.473 & 0.336 & 3.980 & 0.626 & 59.718 \\
\hline 57 & Brussels & 2.671 & 0.340 & 0.306 & 0.722 & 50.064 \\
\hline
\end{tabular}




\section{Appendix B: Mid-Year adjustments of budget targets}

In this subsection, we allow for an adjustment of targets during the planning period. This extension models the situation in practice in which targets are updated during the year. For example, the ICRC annual report 2017 shows that 12 delegations out of 57 had budget amendments.

To study the impact of this flexibility, we assume that the IHO has the possibility to modify the initial plan once d ring he year, e.g., atter $6 \mathrm{~m}$ ohths, for some or all delegations. In other words, at the beginning of the year the IHO determines the initial budget targets, $\boldsymbol{x}$. Then, depending on donations received until then, the IHO may decide to update the initial targets and we denote the updated targets by vector $\mathfrak{x}$. We allow a maximum deviation of the updated targets from the initial targets of $\left(1-\gamma_{i}\right) x_{i} \leq \mathfrak{x}_{i} \leq\left(1+\gamma_{i}\right) x_{i}$, where $0 \leq \gamma_{i}<1$ is a parameter of the maximum allowed deviation from the initial target of delegation $i$. Note that we allow updating in both directions, that is, increasing and decreasing targets.

Let us denote the donations received in the first and in the second half of the year by vectors $\dot{\boldsymbol{\xi}}$ and $\ddot{\boldsymbol{\xi}}$, respectively. The problem can be written as a three stage stochastic program as follows:

$$
\begin{array}{lll}
\text { Stage 1: } & \max _{\boldsymbol{x} \geq 0} \mathfrak{Q}(\boldsymbol{x})=\mathbb{E}_{\tilde{\boldsymbol{\xi}}}\left[Q^{1}(\boldsymbol{x}, \dot{\boldsymbol{\xi}})\right] & \\
\text { Stage 2: } & Q^{1}(\boldsymbol{x}, \dot{\boldsymbol{\xi}})=\max _{\mathfrak{x} \geq 0} \mathbb{E}_{\tilde{\boldsymbol{\xi}} \mid \dot{\boldsymbol{\xi}}}\left[Q^{2}(\mathfrak{x}, \dot{\boldsymbol{\xi}}+\ddot{\boldsymbol{\xi}})\right] & \\
& \text { s.t. } \quad x_{i}\left(1-\gamma_{i}\right) \leq \mathfrak{x}_{i} \leq x_{i}\left(1+\gamma_{i}\right), \quad \forall i \in I
\end{array}
$$

Stage 3:

$$
\begin{aligned}
& Q^{2}(\mathfrak{x}, \dot{\boldsymbol{\xi}}+\ddot{\boldsymbol{\xi}})=\max _{\boldsymbol{y} \geq 0} U(\mathfrak{x}, \dot{\boldsymbol{\xi}}+\ddot{\boldsymbol{\xi}}, \boldsymbol{y}) \\
& \text { s.t. } \quad \sum_{i=1}^{n} y_{i} \leq \dot{\xi}_{n e}+\ddot{\xi}_{n e}
\end{aligned}
$$

In the above model, the $\mathrm{IHO}$ first decides upon $\boldsymbol{x}$, knowing the distribution of donations in the first and second half of the year (Stage 1). Then, in the middle of the year, based on the observed donations from the first period, $\mathfrak{x}$ is chosen to optimize $Q^{1}(\mathfrak{x}, \dot{\boldsymbol{\xi}}, \ddot{\boldsymbol{\xi}}$ ) (Stage 2). Finally, at the end of the year, the nonearmarked donations are allocated to the different delegations in order to maximize the IHO's overall utility, $U(\mathfrak{x}, \dot{\boldsymbol{\xi}}+\ddot{\boldsymbol{\xi}}, \boldsymbol{y})$ (Stage 3). A similar logic could be applied to further extend the model to multiple adjustments of the budget targets.

In Model (B.1), if $\gamma_{i}=0$ for all $i$ and $B(\boldsymbol{\xi})=B(\dot{\boldsymbol{\xi}}+\ddot{\boldsymbol{\xi}}), \mathfrak{x}_{i}=x_{i}$, and the results will be the same as the model that we presented in Section 3. As $\gamma_{i}$ increases, the feasible space does not decrease, and thus, the maximum IHO utility is non decreasing in $\gamma_{i}$.

Developing an efficient solution method for this 3-stage model is out of the scope of this paper. We therefore solve it using its extensive form. By discretizing the uncertainty into a limited number of scenarios, the model can be written as follows:

$$
\begin{aligned}
\max _{\boldsymbol{x}, \mathfrak{x}, \boldsymbol{\alpha}, \boldsymbol{y}} \overline{\mathfrak{Q}}(\boldsymbol{x}, \mathfrak{x}, \boldsymbol{\alpha}, \boldsymbol{y})= & \sum_{\dot{k} \in \dot{K}} \sum_{\ddot{k} \in \ddot{K}(\dot{k})} p_{\dot{k}} p_{\ddot{k}} \sum_{i=1}^{n}\left[f_{i}\left(\alpha_{i}^{\dot{k} \ddot{k}}\right)-g_{i}\left(\mathfrak{x}_{i}^{\dot{k}}-\alpha_{i}^{\dot{k} \ddot{k}}\right)\right] \\
\text { s.t. } \quad & \mathfrak{x}_{i}^{\dot{k}} \leq x_{i}\left(1+\gamma_{i}\right) \\
& x_{i}\left(1-\gamma_{i}\right) \leq \mathfrak{x}_{i}^{\dot{k}} \\
& \left.\alpha_{i}^{\dot{k}}\right] \leq \mathfrak{x}_{i}^{\dot{k}}
\end{aligned}
$$

$$
\begin{aligned}
& \dot{k} \in \dot{K}, i \in I \\
& \dot{k} \in \dot{K}, i \in I \\
& \dot{k} \in \dot{K}, \ddot{k} \in \ddot{K}(\dot{k}), i \in I
\end{aligned}
$$


Keshvari Fard, Ljubić, and Papier: Budgeting in International Humanitarian Organizations

$$
\begin{array}{ll}
\alpha_{i}^{\dot{k} \ddot{k}} \leq \xi_{i}^{\dot{k}}+\xi_{i}^{\ddot{k}}+y_{i}^{\dot{k} \ddot{k}} & \dot{k} \in \dot{K}, \ddot{k} \in \ddot{K}(\dot{k}), i \in I \\
\sum_{i=1}^{n} y_{i}^{\dot{k} \ddot{k}} \leq \xi_{n e}^{\dot{k}}+\xi_{n e}^{\ddot{k}} & \dot{k} \in \dot{K}, \ddot{k} \in \ddot{K}(\dot{k}) \\
x_{i}, \mathfrak{x}_{i}^{\dot{k}}, y_{i}^{\dot{k} \ddot{k}}, \alpha_{i}^{\dot{k} \ddot{k}} \in \mathbb{R}^{+} & \dot{k} \in \dot{K}, \ddot{k} \in \ddot{K}(\dot{k}), i \in I
\end{array}
$$

In this model, $\dot{K}$ represents the set of potential scenarios for donations in the first period, while $\ddot{K}(\dot{k})$ is the set of donation scenarios for the second period if donation $\dot{k}$ is received in the first period. This would allow to model scenarios where donations in the two periods are dependent. $p_{\dot{k}}$ and $p_{\ddot{k}}$ are the probabilities of $\dot{k}^{\text {th }}$ and $\ddot{k}^{\text {th }}$ scenarios in $\dot{K}$ and $\ddot{K}(\dot{k}), \mathfrak{x}_{i}^{\dot{k}}$ is the revised target for delegation $i$ after observing $\boldsymbol{\xi}^{\dot{k}}, \alpha_{i}^{\dot{k} \ddot{k}}$ and $y_{i}^{\dot{k} \ddot{k}}$ are the budget spent by delegation $i$, and the nonearmarked budget allocated to delegation $i$ under realizations $\dot{k}$ and $\ddot{k}$, respectively. The objective function is concave, so the optimal value for $\mathfrak{x}, \boldsymbol{\alpha}$ and $\boldsymbol{y}$ can be found.

Solving the above model is computationally intensive; hence, we can only compute the optimal solution for the case of $n=2$ and $n=3$ delegations and with $|\dot{K}|=|\ddot{K}|=25$ (equivalent to 625 scenario combinations). We assume that the deviation threshold is the same for all delegations, i.e., $\gamma_{i}=\gamma$ for all $i$ and that $B(\dot{\boldsymbol{\xi}})$ and $B(\ddot{\boldsymbol{\xi}})$ are i.i.d. normal distributions such that $\mathbb{E}(\dot{\boldsymbol{\xi}})=\mathbb{E}(\ddot{\boldsymbol{\xi}})=0.5 \mathbb{E}(\boldsymbol{\xi})$ and $\boldsymbol{\sigma}^{2}(\dot{\boldsymbol{\xi}})=\boldsymbol{\sigma}^{2}(\ddot{\boldsymbol{\xi}})=0.5 \boldsymbol{\sigma}^{2}(\boldsymbol{\xi})$.

Figure B.1 shows the numerical results for different values of $\gamma$. For $n=2$ delegations, our findings indicate that by increasing deviation threshold $\gamma$ from 0 to 0.2 , IHO utility increases by $8.9 \%$, beneficiaries' welfare by $4.3 \%$, with $2.8 \%$ higher final budget targets $\mathfrak{x}$. For $n=3$ delegations, we obtain $5.8 \%$ more IHO utility, $3.2 \%$ higher welfare with $3.2 \%$ greater final targets.

It is interesting to note that the flexibility to adjust leads to on average higher targets in our numerical experiments. A possible explanation may be that the reduced variability at the time of adjustment requires less protection against the penalty for unmet targets and hence allows for updating the targets upwards; similar effects have been observed before in the literature on allocation problems; see, e.g., Papier (2016).

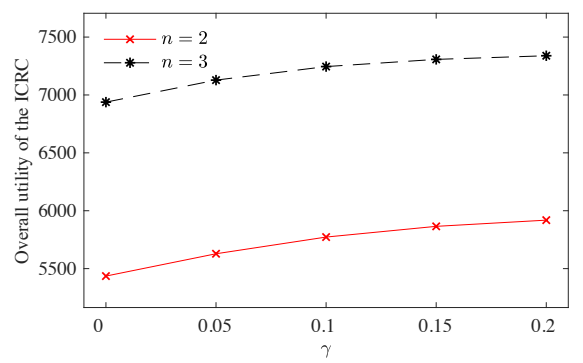

(a) Overall utility of the IHO

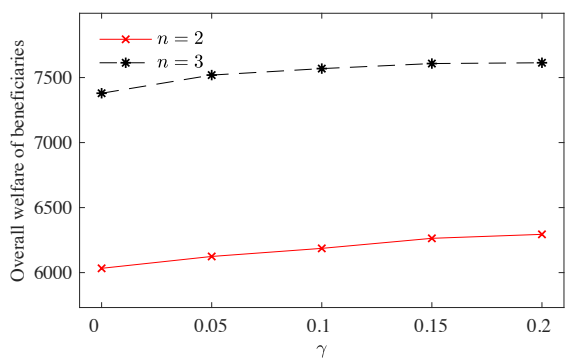

(b) Overall welfare of beneficiaries

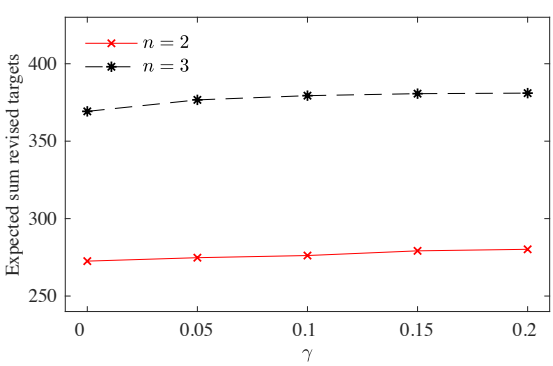

(c) Sum of budget targets

Figure B.1 The IHO utility, beneficiaries' welfare, and final targets when allowing updating budget targets in the middle of the year for two different sets of delegations. 


\section{Appendix C: Numerical procedure for the target-dependent distribution of donations}

In this appendix we propose a simple procedure based on Nelder-Mead Simplex algorithm to solve generic Model (17) presented in Section 6. By discretizing the donations, for any given $\boldsymbol{x}, \mathscr{Q}(\boldsymbol{x})$ can be written as follows:

$$
\begin{aligned}
& \overline{\mathscr{Q}}(\boldsymbol{x})=\max _{\boldsymbol{\alpha}, \boldsymbol{y}} \sum_{k \in K_{\boldsymbol{x}}}\left[\mathbb{P}\left(\boldsymbol{\xi}^{k} \mid \boldsymbol{x}\right) \sum_{i=1}^{n}\left[f_{i}\left(\alpha_{i}^{k}\right)-g_{i}\left(x_{i}-\alpha_{i}^{k}\right)\right]\right] \\
& \alpha_{i}^{k} \leq x_{i} \\
& \alpha_{i}^{k} \leq \xi_{i}^{k}+y_{i}^{k} \\
& \sum_{i=1}^{n} y_{i}^{k} \leq \xi_{n e}^{k},
\end{aligned}
$$

$$
\begin{aligned}
& k \in K_{\boldsymbol{x}}, i \in I \\
& k \in K_{\boldsymbol{x}}, i \in I \\
& k \in K_{\boldsymbol{x}}
\end{aligned}
$$

where $K_{\boldsymbol{x}}$ is the discretized set of scenarios under budget target $\boldsymbol{x}$. The model is feasible for any $\boldsymbol{x} \geq 0$, and the feasible space is a convex set. The objective function is jointly concave in $\boldsymbol{\alpha}$ and $\boldsymbol{y}$; therefore, Model (C.1) can be easily solved for any given $\boldsymbol{x}$ and $K_{\boldsymbol{x}}$. However, because the probability distribution of donations depends upon the target decisions, $\overline{\mathscr{Q}}(\boldsymbol{x})$ is not concave in $\boldsymbol{x}$ (see, for example, Hellemo et al. (2018)), and thus, we cannot use standard convex optimization techniques, such as the interior point method, to find the optimal value of $\boldsymbol{x}$.

A strong optimization heuristic that is frequently used for nonconvex optimization is the Nelder-Mead simplex algorithm (Nelder and Mead 1965), although this method does not guarantee finding the global optimum. We implemented the Nelder-Mead simplex algorithm by using the standard parameter values. For each new set of budget targets $\boldsymbol{x}$, we identified the distribution of all $\xi_{i}$ and generated $\left|K_{\boldsymbol{x}}\right|$ realizations accordingly. Model (C.1) was then solved to find $\overline{\mathscr{Q}}(\boldsymbol{x})$. The Nelder-Mead simplex algorithm continued to find new $\boldsymbol{x}$ and updated the basis, until the terminating condition was met.

\section{Appendix D: Systematic identification of utility function parameters}

In this section, we present a systematic estimation tool for the three parameters of the utility function that capture a delegation's characteristic in our model, $a_{i}^{f}, b_{i}^{f}$, and $a_{i}^{g}$.

To derive the welfare gains of each delegation, the decision maker first has to identify the different operations that a delegation can perform as well as their impact on the beneficiaries' welfare. This impact may comprise factors such as the nature of the operation, the number of people affected, localities, and target groups (Mullen 1995).

Consequently, multicriteria optimization models have been developed for resource allocation in healthcare and humanitarian operations. These models require the relative weights of each criteria as a basis for determining the relative impact or importance of each operation. Methods to elicit these weights using the stated preferences of the IHO managers and other field experts include conjoint analysis (Orme 2010), contingent valuation (Holguín-Veras et al. 2016), and discrete choice experiments (de Bekker-Grob et al. 2012). One can refer to Mullen and Spurgeon (2018) for an overview of additional techniques as well as a detailed discussion of priority evaluation. 
For each operation of a delegation, the $\sqrt{\mathrm{HO}}$ estimates the impat on the welfare of beneficiaries and the cost of the operation. The operations are sorted by decreasing welfape per eost unit and plotted in a cumulative welfare chart. A power function is fitted to the plotted welfare function to estimate parameters $a_{i}^{f}$ and $b_{i}^{f}$ of the delegation.

We illustrate the above steps with an example, which is based on data of the count ies of South Sudan and Somolia from tho ICRC Annual Report 2017 where possible. In our example, an IHO performs two operations, food commodities and water and habitat activities by two delegations in two different countries, A and B. The IHO prioritizes operations based on three factors: the number of beneficiaries being served $(N)$, the importance of an operation $\left(W_{j}^{f}\right)$, and the criticality of a location $\left(V_{i}^{f}\right)$.

Using conjoint analysis, the IHO managers conclude that the importance of food commodities is twice as high as the importance of water and habitat activities for an average beneficiary, and thus, $W_{j}^{f} \in\{2,1\}$, where $j=1$ and $j=2$ refer to food commodities and water and habitat activities. resp etivel . Similarly, due to civil war in the territory of delegation A, the IHO puts a higher weight on the operations in this delegation and thus $W_{i}^{f} \in\{2,1.5\}$, with $i=1$ and $i=2$ refer to delegation A and B. respectivelv.

Finally, the IHO uses a utility function where the utility of performing operation $j$ for $N$ ventertiaries in delegation $i$ is computed as $u(i, j, N)=N W_{j}^{f} W_{i}^{f}$. In other cases, the prioritization can be more complex; for example, ICRC distinguishes the beneficiaries into men, women, and children.

Next, delegations and the top IHO management estimate the costs of each operation. Similar to Gralla et al. (2014), Jana et al. (2019), the IHO considers different levels of demand fulfillment, e.g., 40\%, 60\% and $80 \%$ coverage of all potential beneficiaries in the territory of each delesation. The territory of delegation $B$ has a long coastal line with functioning ports and lower dispersion of demand points. The currency exchange rate and purchasing power parity of $\mathrm{B}$ is low as well, which reduces the costs of operations. On the other hand, the logistics costs of operations of delegation A are higher due to lack of access to the sea, destruction of roads during the civil war, geographical dispersion of demand points, and higher risks involved in conductins missions. Figure D.1 shows the map of these countries.

In general, one can expect that the densely populated areas near the IHO centers are served first, as they pose less logistical needs with faster and more efficient demand fulfilment. The more distant and dispersed locations lead to higher cost to serve per beneficiary, given the larger number of trips, longer travel times, and higher risks involved. Therefore, the cost per beneficiary rises as the coverage level increases. In this example, large, medium and small demand points are served at 40\%,60\% and 80\% coverage, respectively.

Table D.1 summarizes the required data to determ he the beneficiaries' welf re function for delegations A and B. The last column contains the additional impact per unit cost spent on each operation. To achieve efficiency and effectiveness, the IHO prioritizes the operations w.r.t. this ratio.

Figure D.2 shows the cumulative utility for each country as a fumcion of the pudget spent. We use a trustregion algorithm (see Helfrich and Zwick (1996), to fit a power function $u_{i}\left(\alpha_{i}\right)=a_{i}^{f} \alpha_{i}^{b_{i}^{f}}$ to each delegation. The fitted functions are shown in Figure D.2. We optain $u_{1}-450.70$ aाlu $u_{2}^{f}+1007.73$, and $b_{1}^{f}=0.55$ and $b_{2}^{f}=0.51$.

The IHO also uses stated preferenee teehniques to determine the demand mismatch penalty factor $a_{i}^{g}$ for each delegation $i$. The factors that can contribute to the preferences an affect the pena. fy factor may include 


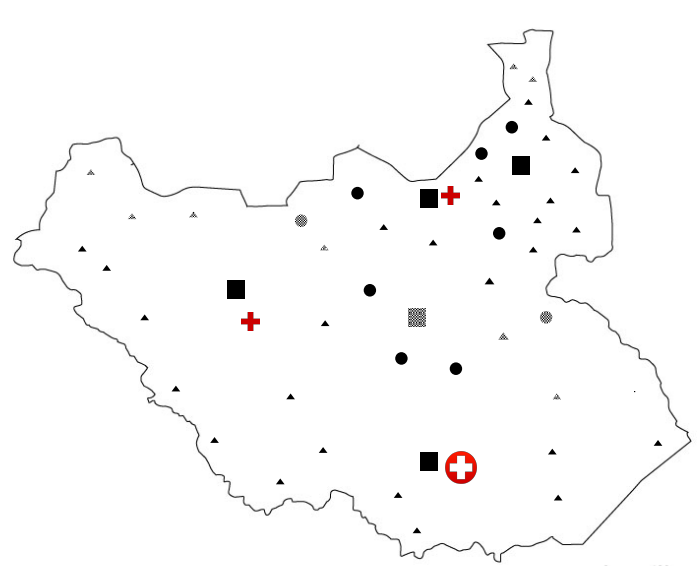

Country A

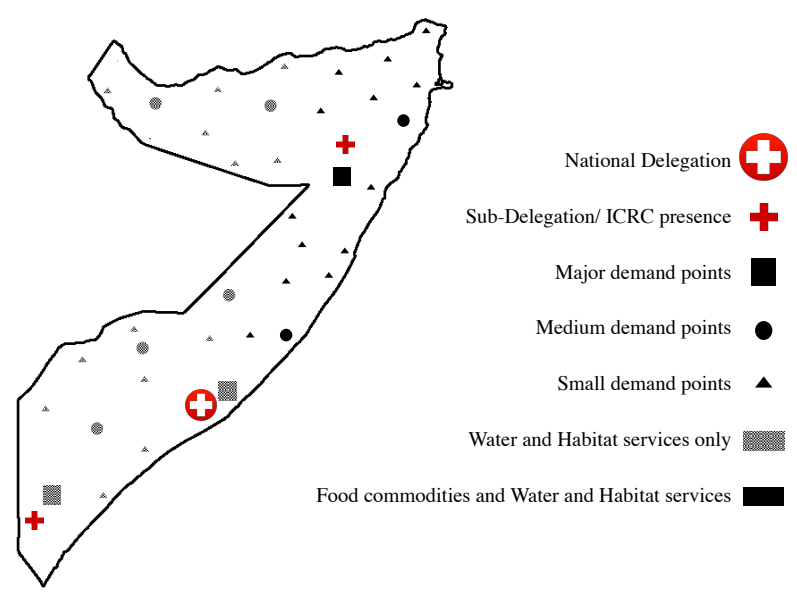

Country B

Figure D.1 Dispersion of demand points in the territories of delegations $A$ and $B$.

Table D.1 Information about operations for delegations A and B

\begin{tabular}{|c|c|c|c|c|c|c|c|c|c|}
\hline Country & $\begin{array}{l}\text { Operation } \\
\text { code }\end{array}$ & Operation Name & $\begin{array}{l}\text { Cumulative } \\
\text { coverage }\end{array}$ & $\begin{array}{l}\text { Population } \\
\text { served }\end{array}$ & $\begin{array}{l}\text { Operation } \\
\text { Weight }\end{array}$ & $\begin{array}{l}\text { Location } \\
\text { Weight }\end{array}$ & Impact & $\begin{array}{l}\text { Cost } \\
\text { (KCHF) }\end{array}$ & $\begin{array}{l}\text { Impact/ } \\
\text { cost ratio }\end{array}$ \\
\hline \multirow{6}{*}{$\mathrm{A}$} & $\mathrm{AF} 40$ & Food Commodities & $40 \%$ & 132,000 & 2 & 2 & 528,000 & 588600 & 0.897 \\
\hline & AF60 & Food Commodities & $60 \%$ & 66,000 & 2 & 2 & 264,000 & 501400 & 0.527 \\
\hline & $\mathrm{AF} 80$ & Food Commodities & $80 \%$ & 66,000 & 2 & 2 & 264,000 & 1090000 & 0.242 \\
\hline & AW40 & Water and habitat activities & $40 \%$ & 156,000 & 1 & 2 & 312,000 & 264500 & 1.180 \\
\hline & AW60 & Water and habitat activities & $60 \%$ & 78,000 & 1 & 2 & 156,000 & 264500 & 0.590 \\
\hline & AW80 & Water and habitat activities & $80 \%$ & 78,000 & 1 & 2 & 156,000 & 621000 & 0.251 \\
\hline \multirow{6}{*}{ B } & BF40 & Food Commodities & $40 \%$ & 180,000 & 2 & 1.5 & 540,000 & 940500 & 0.574 \\
\hline & BF60 & Food Commodities & $60 \%$ & 90,000 & 2 & 1.5 & 270,000 & 855000 & 0.316 \\
\hline & BF80 & Food Commodities & $80 \%$ & 90,000 & 2 & 1.5 & 270,000 & 1054500 & 0.256 \\
\hline & BW40 & Water and habitat activities & $40 \%$ & 444,000 & 1 & 1.5 & 666,000 & 347500 & 1.917 \\
\hline & BW60 & Water and habitat activities & $60 \%$ & 222,000 & 1 & 1.5 & 333,000 & 417000 & 0.799 \\
\hline & BW80 & Water and habitat activities & $80 \%$ & 222,000 & 1 & 1.5 & 333,000 & 625500 & 0.532 \\
\hline
\end{tabular}

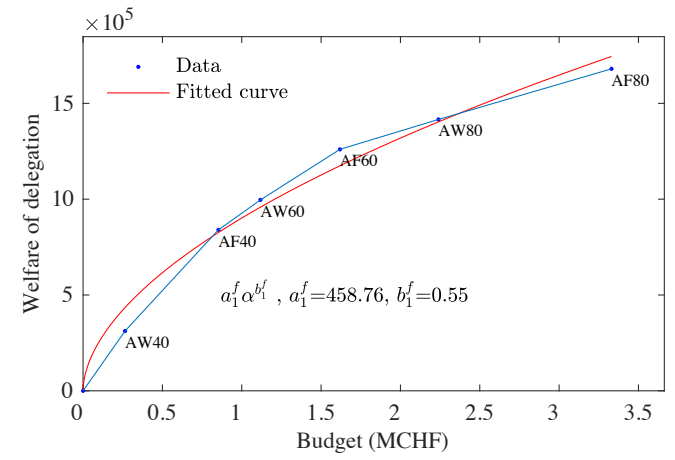

(a) Delegation A

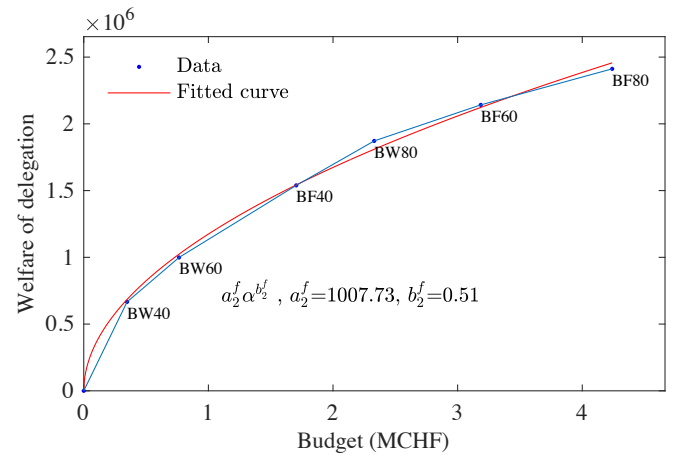

(b) Delegation B

Figure D.2 Estimated data and fitted function for utility as a function of budget.

but are not limited to the delegation's population, population in need of aid, number of donors, their type (e.g., governments or corporations), their importance and size of their donations, geopolitical importance of the country, media coverage, and the presence of other NGOs in the region. These factors affect the IHO's reputation and visibility as well as its access to future fundraising for that region. The penalty scores 
Keshvari Fard, Ljubić, and Papier: Budgeting in International Humanitarian Organizations

Manufacturing \& Service Operations Management 00(0), pp. 000-000, C) 0000 INFORMS

Table D.2 Summary of the systematic estimation approach.

\begin{tabular}{|c|c|c|c|}
\hline No. & Step & Description & Potential methods \\
\hline 1 & $\begin{array}{l}\text { Identification of mis- } \\
\text { sions of each delega- } \\
\text { tion }\end{array}$ & $\begin{array}{l}\text { Identification of all relev } \\
\text { that a delegation could per }\end{array}$ & $\begin{array}{l}\text { Interviews/requests from local and } \\
\text { regional managers, local authorities, } \\
\text { etc. }\end{array}$ \\
\hline 2 & $\begin{array}{l}\text { Estimation of the } \\
\text { impact of each mission }\end{array}$ & $\begin{array}{l}\text { Identification of impact criteria, elicita- } \\
\text { tion of criteria weights, estimation of } \\
\text { impact for each mission on each criterion }\end{array}$ & $\begin{array}{l}\text { Stated preferences technique with } \\
\text { conjoint analysis, contingent valua- } \\
\text { tion, or discrete choice experiments }\end{array}$ \\
\hline 3 & $\begin{array}{l}\text { Estimation of the cost } \\
\text { of each mission }\end{array}$ & $\begin{array}{l}\text { Calculation of the expected cost of each } \\
\text { operation, given the population covered, } \\
\text { logistical expenses, personnel cost, etc. }\end{array}$ & $\begin{array}{l}\text { Expert interviews, historical data, } \\
\text { statistical methods }\end{array}$ \\
\hline 4 & $\begin{array}{l}\text { Derivation of welfare } \\
\text { function of each dele- } \\
\text { gation }\end{array}$ & $\begin{array}{l}\text { Sorting missions by decreasing welfare } \\
\text { per unit cost, fitting power function to } \\
\text { the data }\end{array}$ & $\begin{array}{l}\text { Curve fitting approaches such as } \\
\text { Trust region algorithm }\end{array}$ \\
\hline 5 & $\begin{array}{l}\text { Estimation of mis- } \\
\text { match penalty cost } \\
\text { factor }\end{array}$ & $\begin{array}{l}\text { Identification of relative weight of mis- } \\
\text { match penalty for different delegations, } \\
\text { normalization of the factors to same scale } \\
\text { as welfare function }\end{array}$ & $\begin{array}{l}\text { Expert interviews, Stated prefer- } \\
\text { ences technique }\end{array}$ \\
\hline
\end{tabular}

allocated to each delegation are then normalized to the scale of the beneficiaries' welfare function to make them comparable.

For our example, we assume that the IHO considers three main factors: number of active IHOs in the country $\left(W_{1}^{g}\right)$, overall population $\left(W_{2}^{g}\right)$ and the number of followers of the countries' WHO twitter account $\left(W_{3}^{g}\right)$. For delegation A, these numbers are $37,10.98 \mathrm{M}$ and $6.1 \mathrm{~K}$, while for delegation $\mathrm{B}$, these numbers are $74,15.1 \mathrm{M}$ and $41 \mathrm{~K}$. Assuming the IHO computes the penalty factors using $3 \ln \left(W_{1}^{g}\right)+2 \ln \left(W_{2}^{g}\right)+1 \ln \left(W_{3}^{g}\right)$, the unnormalized penalty factors are obtained as 52.0 for delegation A and 56.6 for delegation $\mathrm{B}$. Using an approach similar to that in Section 5.1 to normalize the penalty function w.r.t. the welfare function (setting $\max _{i} a_{i}^{f}=\max _{i} a_{i}^{g}$ ), we obtain $a_{1}^{g}=925.4$ and $a_{2}^{g}=1007.7$.

The approach that we have illustrated above with an example can be used for systematic estimation of the function parameters. Table D.2 summarizes the key steps of this approach.

\section{References}

de Bekker-Grob, E. W., Ryan, M., and Gerard, K. (2012). Discrete choice experiments in health economics: a review of the literature. Health economics, 21(2):145-172.

Gralla, E., Goentzel, J., and Fine, C. (2014). Assessing trade-offs among multiple objectives for humanitarian aid delivery using expert preferences. Production and Operations Management, 23(6):978-989.

Helfrich, H.-P. and Zwick, D. (1996). A trust region algorithm for parametric curve and surface fitting. Journal of computational and applied mathematics, 73(1-2):119-134.

Hellemo, L., Barton, P. I., and Tomasgard, A. (2018). Decision-dependent probabilities in stochastic programs with recourse. Computational Management Science, 15(3-4):369-395.

Holguín-Veras, J., Amaya-Leal, J., Cantillo, V., Van Wassenhove, L. N., Aros-Vera, F., and Jaller, M. (2016). Econometric estimation of deprivation cost functions: A contingent valuation experiment. Journal of Operations Management, 45:44-56. 
Jana, R. K., Chandra, C. P., and Tiwari, A. K. (2019). Humanitarian aid delivery decisions during the early recovery phase of disaster using a discrete choice multi-attribute value method. Annals of Operations Research, 283(1):1211-1225.

Mullen, P. (1995). Priority setting in health care: Techniques and pitfalls. Managing health care under resource constraints, page 105.

Mullen, P. and Spurgeon, P. (2018). Priority setting and the public. CRC Press.

Nelder, J. A. and Mead, R. (1965). A simplex method for function minimization. The computer journal, $7(4): 308-313$.

Orme, B. (2010). Getting started with conjoint analysis: strategies for product design and pricing research second edition. Madison: Research Publishers LLC.

Papier, F. (2016). Supply allocation under sequential advance demand information. Operations Research, $64(2): 341-361$. 


\section{Supplementary Appendix for Budgeting in International Humanitarian Organizations}

\section{Appendix E: Additional numerical experiments}

In this section, we first present comparison of computational time for GBD, GBD-g and extensive-form formulations for small size problems. We then present two additional numerical experiments: the sensitivity of the model with respect to changes in the parameters of the utility functions and the impact of budget uncertainty.

Comparison of computational time: As we can see in Table E.1, the proposed GBD algorithm significantly outperforms the other two alternatives. We focus on smaller instances $(n \leq 15)$ that can be addressed by all three methods. We observe that the extensive form scales very poorly with the increasing size of $n$ and $|K|$.

Table E.1 Comparison of the required computational time (in seconds) by the new proposed GBD algorithm, a GBD algorithm using general-purpose nonlinear solver (GBD-g), and the extensive form (Ex-form).

\begin{tabular}{lccc|ccc|cccc}
\hline & \multicolumn{3}{c}{$\mathbf{n}=\mathbf{5}$} & \multicolumn{3}{c}{$\mathbf{n}=\mathbf{1 0}$} & \multicolumn{3}{c}{$\mathbf{n}=\mathbf{1 5}$} \\
\cline { 2 - 10 } Sample size & GBD & GBD-g & Ex-form & GBD & GBD-g & Ex-form & GBD & GBD-g & Ex-form \\
\hline$|\mathbf{K}|=\mathbf{2 5}$ & 0.5 & 1.0 & 2.2 & 1.0 & 4.9 & 13.3 & 1.9 & 9.2 & 55.0 \\
$\mathbf{K} \mid=\mathbf{5 0}$ & 0.9 & 2.0 & 28.0 & 1.8 & 6.2 & 154.1 & 2.6 & 10.4 & 396.5 \\
$\mathbf{K} \mid=\mathbf{7 5}$ & 1.4 & 3.5 & 88.1 & 2.8 & 9.0 & 837.6 & 4.7 & 23.6 & 1997.7 \\
$\mathbf{K} \mid=\mathbf{1 0 0}$ & 1.8 & 4.4 & 169.2 & 4.2 & 18.5 & 1864.8 & 5.0 & 17.1 & 7486.9 \\
\hline
\end{tabular}

Sensitivity analysis of the parameters of the utility functions: In addition to Proposition 2, which describes the behavior of budget targets with respect to variations in $\boldsymbol{a}^{f}, \boldsymbol{b}^{f}$ and $\boldsymbol{a}^{g}$, we performed numerical experiments of these changes to quantify the magnitude of the impact.

Figure E. 1 shows the case where all $a_{i}^{f}$ are changed by $\pm 50 \%$, while other parameters are held fixed at their default values. For $n=30$, a $50 \%$ increase in $\boldsymbol{a}^{f}$ results in a $1.9 \%$ increase in total budget targets, while a $50 \%$ decrease leads to a $2.7 \%$ decrease in the targets.

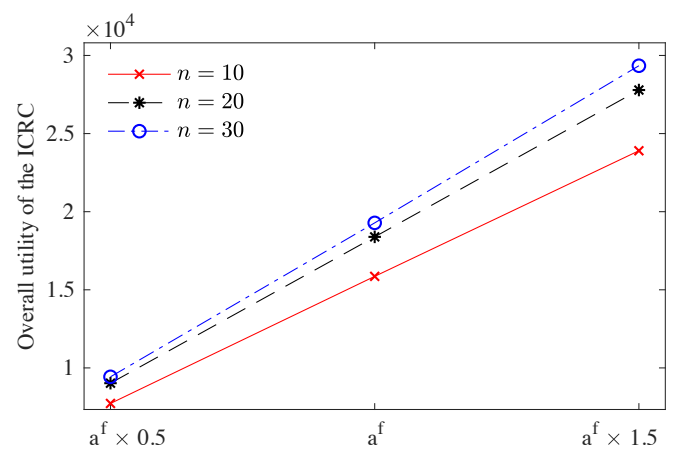

(a) Overall utility of the IHO

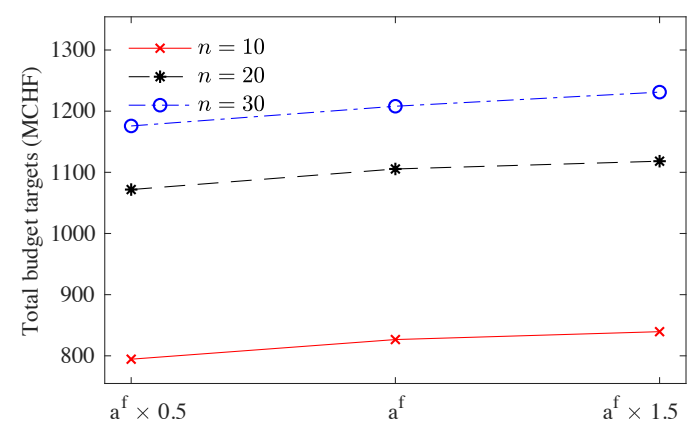

(b) Sum of Budget targets

Figure E.1 The impact of variation in $a^{f}$ : (a) IHO's utility, (b) budget targets.

Increasing and decreasing $\boldsymbol{a}^{g}$ by $50 \%$ while keeping all other parameters fixed results in a $3.3 \%$ decrease and a $2.2 \%$ increase in budget targets, respectively $(n=30)$, which can be observed in Figure E.2. 


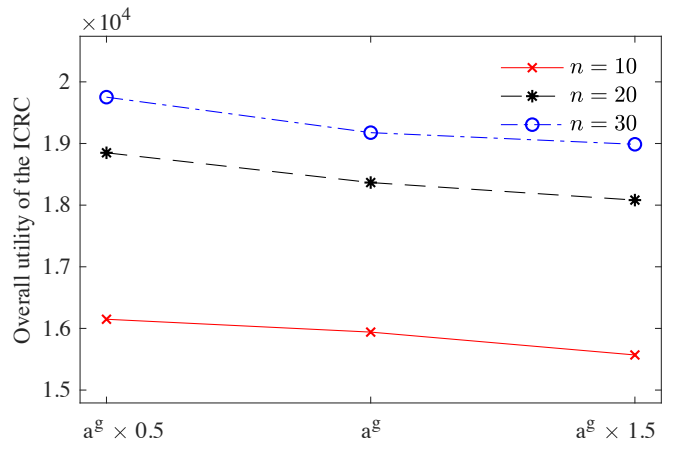

(a) Overall utility of the IHO

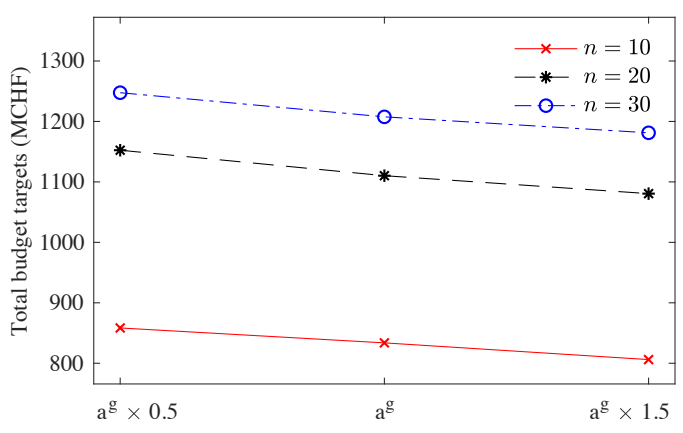

(b) Sum of Budget targets

Figure E.2 The impact of variation in $a^{g}$ : (a) IHO's utility, (b) budget targets.

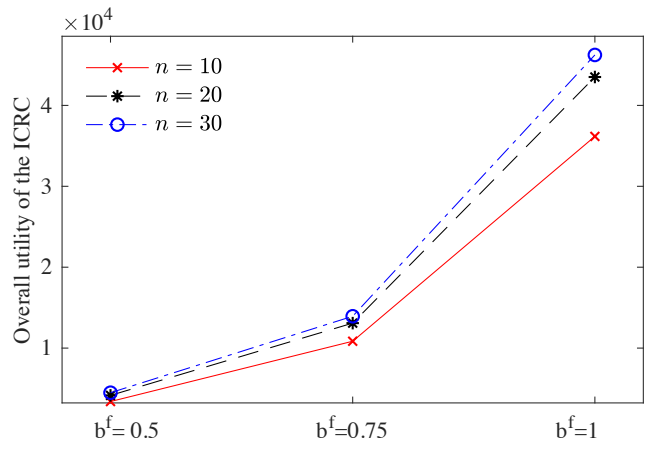

(a) Overall utility of the IHO

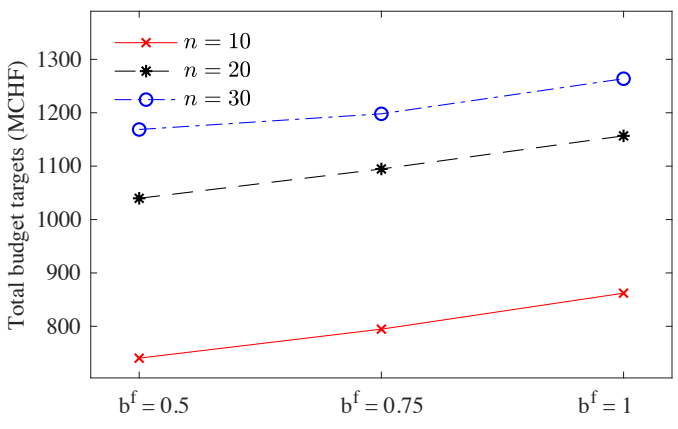

(b) Sum of Budget targets

Figure E.3 The impact of variation in $b^{f}$ : (a) IHO's utility, (b) budget targets.

To investigate the impact of variation in $\boldsymbol{b}^{f}$, we slightly changed our experiments, since increasing $\boldsymbol{b}^{f}$ by $50 \%$ would result in convex beneficiaries' welfare functions for some delegations. Therefore, we fix all $\boldsymbol{b}^{f}$ at three different values, 0.5, 0.75 and 1.0. Note that the latter value leads to linear beneficiaries' welfare functions. From Figure E.3, we can see that by decreasing $b_{i}^{f}$ from 0.75 to 0.5 for all $i(n=30)$, targets decrease by $2.5 \%$, while increasing all $b_{i}^{f}$ to 1 (linear functions) leads to a $5.5 \%$ increase in budget targets.

Impact of budget uncertainty: We investigate the impact of budget uncertainty on the IHO's performance by changing the standard deviation of $\boldsymbol{\xi}$. We consider three settings for the change in all donations (earmarked and nonearmarked), namely, 50\% decrease, no change, and 50\% increase in the standard deviations. We solve the problem using our proposed GBD algorithm.

As we can see in Figure E.4a, high degrees of uncertainty force the IHO to set lower budget targets because decision makers are less certain about receiving donations in the future to fulfill their initial plans, which leads to a lower overall welfare for beneficiaries (Figure E.4b). The risk of failing to meet planned targets due to the additional uncertainty in the budget leads to a comparably sharp decrease in the IHO's utility. Moreover, we can observe that such an impact is independent of the number of delegations that the IHO serves. 


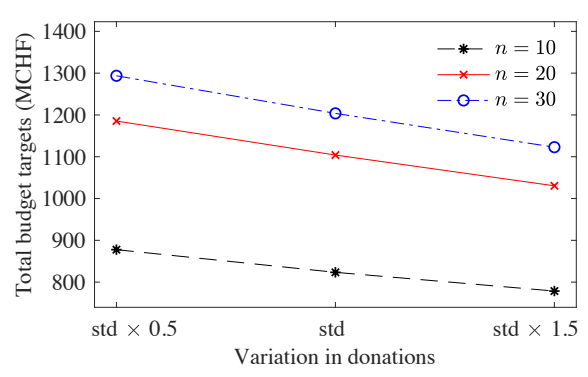

(a) Sum of budget targets

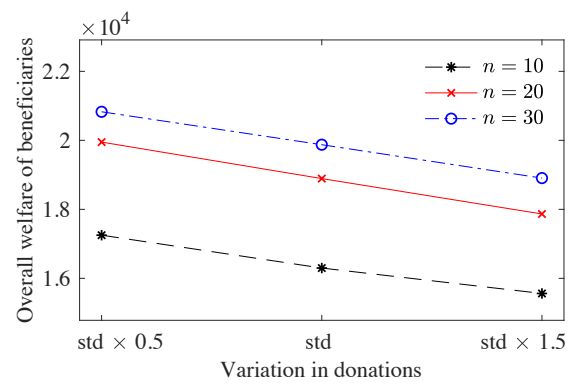

(b) Overall welfare of beneficiaries

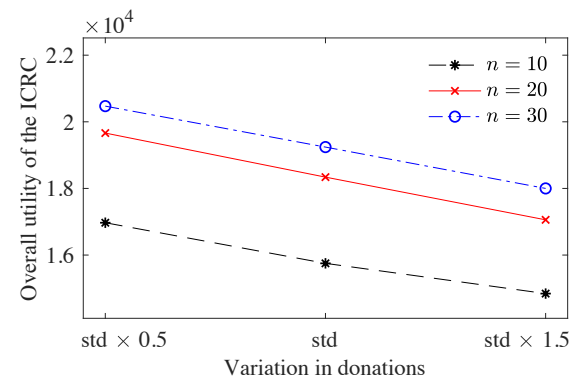

(c) Overall utility of the IHO

Figure E.4 The impact of uncertainty in donations on (a) budget targets, (b) beneficiaries' welfare, and (c) IHO's utility.

Our results show an average increase of $7.1 \%$ in the average targets when reducing the uncertainty by $50 \%$ compared to an average decrease of $6.3 \%$ when increasing uncertainty by $50 \%$. The IHO's utility increases by $5.4 \%$ and welfare by $7.1 \%$ when reducing uncertainty, while utility decreases by $6.4 \%$ and welfare by $4.9 \%$ when uncertainty increases.

Given the above results, measures to reduce uncertainty in donations, such as proposing incentives for donating regularly rather than sporadically, or building online communities could be effective; see, for example, Hart et al. (2005).

\section{Appendix F: Sample Average Approximation algorithm}

We consider replicating the GBD method for $M$ times, with each replication of a size of $|K|$ scenarios. We denote by $\hat{\mathfrak{Q}}_{K}^{m}$ and $\hat{\boldsymbol{x}}_{K}^{m}$ the optimal value and optimal solution of the $\mathrm{m}^{\text {th }}$ SAA replication, $m \in\{1, \ldots, M\}$. An estimator of $\mathfrak{Q}\left(\boldsymbol{x}^{*}\right)$ is given by

$$
\overline{\mathfrak{Q}}_{K}^{M}=\frac{1}{M} \sum_{m=1}^{M} \hat{\mathfrak{Q}}_{K}^{m}
$$

In order to choose among $M$ different solutions of SAA replications, we consider a large sample of size $\left|K^{\prime}\right|>|K|$, and evaluate the expected utility of each solution $\hat{\boldsymbol{x}}_{K}^{m}$ under this new sample. Let us define $\hat{\boldsymbol{x}}$ as the solution that provides the highest utility under the sample of size $\left|K^{\prime}\right|$.

$$
\hat{\boldsymbol{x}}=\arg \max \mathfrak{Q}_{\Omega_{K^{\prime}}}(\boldsymbol{x}), \quad \boldsymbol{x} \in\left\{\hat{\boldsymbol{x}}_{K}^{1}, \ldots, \hat{\boldsymbol{x}}_{K}^{M}\right\}
$$

where $\Omega_{K^{\prime}}$ refers to the set of scenarios in the sample of size $\left|K^{\prime}\right|$, and $\mathfrak{Q}_{\Omega_{K^{\prime}}}(\hat{\boldsymbol{x}})$ is the expected utility of the IHO under $\Omega_{K^{\prime}}$ and $\hat{\boldsymbol{x}}$. Note that $\mathfrak{Q}_{\Omega_{K^{\prime}}}(\hat{\boldsymbol{x}})$ is a stronger estimator of $\mathfrak{Q}\left(\boldsymbol{x}^{*}\right)$ than $\overline{\mathfrak{Q}}_{K}^{M}$. Here, $\hat{\boldsymbol{x}}$ would serve as the optimal solution of the SAA algorithm. We further compute

$$
q_{K}^{m}(\hat{\boldsymbol{x}})=\mathfrak{Q}_{\Omega_{K}^{m}}(\hat{\boldsymbol{x}}), \quad m \in 1, \ldots, M
$$

which refers to the utility of $\hat{\boldsymbol{x}}$ under the scenarios of the $\mathrm{m}^{\text {th }}$ replication of the SAA, $\Omega_{K}^{m}$. We define $\bar{q}_{K}^{M}(\hat{\boldsymbol{x}})=\frac{1}{M} \sum_{m=1}^{M} q_{K}^{m}(\hat{\boldsymbol{x}})$. Now, $\overline{\mathfrak{Q}}_{K}^{M}-\bar{q}_{K}^{M}(\hat{\boldsymbol{x}})$ is an estimator of the optimality gap, $\mathfrak{Q}\left(\boldsymbol{x}^{*}\right)-\mathfrak{Q}_{\Omega_{K^{\prime}}}(\hat{\boldsymbol{x}})$, and its variance, which we refer to as $\check{S}^{2}$ is estimated as

$$
\check{S}^{2}=\frac{1}{M(M-1)} \sum_{m=1}^{M}\left[\left(q_{K}^{m}(\hat{\boldsymbol{x}})-\hat{\mathfrak{Q}}_{K}^{m}\right)-\left(\bar{q}_{K}^{M}(\hat{\boldsymbol{x}})-\overline{\mathfrak{Q}}_{K}^{M}\right)\right]^{2} .
$$




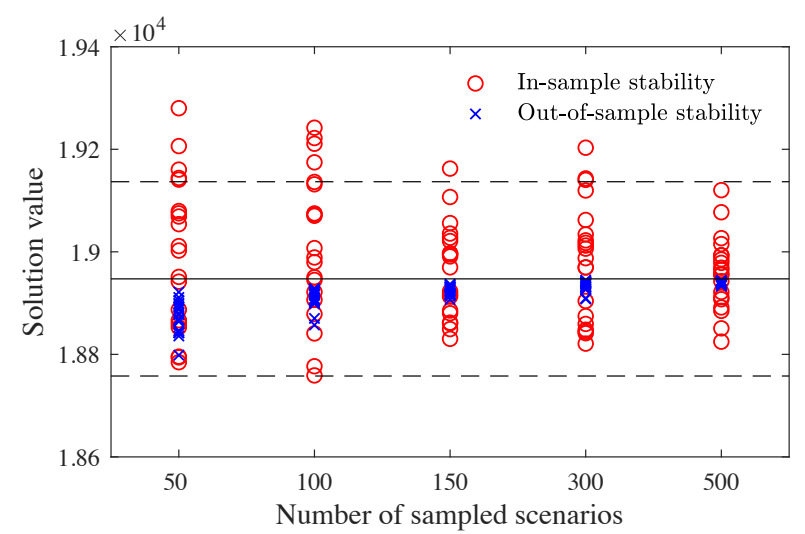

Figure G.1 In- and out-of-sample stability.

To determine the number of required replications, $M$, we start with $M=2$ and continue the process until the $(1-\alpha) \%$ optimality gap (one-sided) is less than the $1 \%$ of the optimal solution, i.e., $\check{S} \times t_{M-1, \alpha} \leq$ $0.01 \mathfrak{Q}_{\Omega_{K^{\prime}}}(\hat{\boldsymbol{x}})$. If this condition is not satisfied until $M$ is reached to a given upper bound, $M^{\text {max }}$, we increase $|K|$ and repeat the process.

\section{Appendix G: Sample stability}

Let $\mathfrak{Q}_{\boldsymbol{S}}(\boldsymbol{x})=\mathbb{E}_{S} Q(\boldsymbol{x}, \boldsymbol{\xi})$, and $\max _{\boldsymbol{x}} \mathfrak{Q}_{\boldsymbol{S}}(\boldsymbol{x})$ denote the stochastic program restricted to a sampled scenario set $\boldsymbol{S}$. Moreover, let $\overline{\boldsymbol{S}}$ and $\hat{\boldsymbol{S}}$ denote two sets of scenarios of the same size, with $\overline{\boldsymbol{x}}$ and $\hat{\boldsymbol{x}}$ optimal solutions to $\max _{\boldsymbol{x}} \mathfrak{Q}_{\bar{S}}(\boldsymbol{x})$ and $\max _{\boldsymbol{x}} \mathfrak{Q}_{\hat{S}}(\boldsymbol{x})$. A scenario generation method is called in-sample stable if the optimal solution values of two independently sampled scenario sets are similar, i.e., if $\mathfrak{Q}_{\overline{\boldsymbol{S}}}(\overline{\boldsymbol{x}}) \approx \mathfrak{Q}_{\hat{\boldsymbol{S}}}(\hat{\boldsymbol{x}})$. On the other hand, if $\mathfrak{Q}_{\check{\boldsymbol{S}}}(\overline{\boldsymbol{x}}) \approx \mathfrak{Q}_{\check{\boldsymbol{S}}}(\hat{\boldsymbol{x}})$ holds for a separate set of scenarios $\check{\boldsymbol{S}}$, the method is called out-of-sample stable. For an in-depth discussion of these concepts, one can refer to King and Wallace (2012).

In order to check for sample stability, we consider the problem with the main $n=25$ ICRC delegations, and find its pseudo-optimal solution using a sample $K^{\prime}$ of size $\left|K^{\prime}\right|=1500$. We then compute in- and outof-sample stability values by sampling subsets of size $|K|$ from $K^{\prime}$. We let this sample size vary between 50 and 500, where for each given size, we sample $M=20$ instances $K_{1}, \ldots, K_{M}$, from $K^{\prime}$. Figure G.1 shows the results of our analysis. The solid horizontal line represents the value of the pseudo-optimal solution i.e., the solution obtained by taking all scenarios in $K^{\prime}$ into account, and the dashed horizontal lines indicate the 99\% confidence interval, i.e., [0.99opt, 1.01opt].

As we can see in Figure G.1, out-of-sample stability can be reached under $|K|=50$ scenarios (i.e., optimal first-stage solutions, evaluated on the whole set of 1500 scenarios, fall within a $99 \%$ of the optimal solution). However, in-sample stability is not reached until $|K| \geq 500$.

\section{Appendix H: Proofs and technical results}

Lemma 3. Following are a number of properties of the problem:

1. For a given budget target $\boldsymbol{x}$ and realization of donations $\boldsymbol{\xi}, U(\boldsymbol{x}, \boldsymbol{\xi}, \boldsymbol{y})$ is concave and non decreasing in $\boldsymbol{y}$.

2. Model (1) is a stochastic program with complete recourse. 
3. $Q(\boldsymbol{x}, \boldsymbol{\xi})$ is non decreasing in $\xi_{n e}$ and $\xi_{i}$, for all $i \in I$.

4. $Q(\boldsymbol{x}, \boldsymbol{\xi})$ is jointly concave in $(\boldsymbol{x}, \boldsymbol{\xi})$, and is an upper semicontinuous function in $\boldsymbol{x}$ for all $\boldsymbol{\xi}$.

5. $\mathfrak{Q}(\boldsymbol{x})$ is concave and upper semicontinuous in $\boldsymbol{x}$.

6. Model (1) has a finite optimal solution and the maximum is attained.

Proof of Lemma 1 For each $i$ that $y_{i}>\left[x_{i}-\xi_{i}\right]^{+}, \min \left\{x_{i}, \xi_{i}+y_{i}\right\}=x_{i}$, and $f_{i}\left(\min \left\{x_{i}, \xi_{i}+y_{i}\right\}\right)=f_{i}\left(x_{i}\right)$. Also, $y_{i}>\left[x_{i}-\xi_{i}\right]^{+} \Longrightarrow\left[x_{i}-\xi_{i}-y_{i}\right]^{+}=0 \Longrightarrow g_{i}\left(\left[x_{i}-\xi_{i}-y_{i}\right]^{+}\right)=g_{i}(0)=0 \Longrightarrow u_{i}\left(x_{i}, \xi_{i}, y_{i}\right)=f_{i}\left(x_{i}\right)$; in other words, for $y_{i}>\left[x_{i}-\xi_{i}\right]^{+}, f_{i}($.$) and g_{i}($.$) would be equal to the case that y_{i}=\left[x_{i}-\xi_{i}\right]^{+}$, and thus in optimality, $y_{i} \leq\left[x_{i}-\xi_{i}\right]^{+}$.

Proof of Proposition 1 Using linearization $\alpha_{i} \leq \min \left\{x_{i}, \xi_{i}+y_{i}\right\}$, the recourse problem for any given pair $(\check{\boldsymbol{x}}, \boldsymbol{\xi})$ can be written as follows.

$$
\begin{aligned}
& Q(\check{\boldsymbol{x}}, \boldsymbol{\xi})=\max _{\boldsymbol{y}, \boldsymbol{\alpha}} \sum_{i \in I}\left[f_{i}\left(\alpha_{i}\right)-g_{i}\left(\check{x}_{i}-\alpha_{i}\right)\right] \\
& \text { s.t. } \alpha_{i} \leq \check{x}_{i}, \quad i \in I \\
& \alpha_{i} \leq \xi_{i}+y_{i}, \quad i \in I \\
& \sum_{i=1}^{n} y_{i} \leq \xi_{n e} \\
& y_{i} \geq 0, \quad i \in I
\end{aligned}
$$

Let $\eta_{i}, \mu_{i}$, and $\mu_{n e}$ and $\rho_{i}$ refer to the dual variables associated with Constraint sets (H.1b), (H.1c), (H.1d) and (H.1e) respectively. The associated Lagrangian function reads as:

$$
L(\check{\boldsymbol{x}}, \boldsymbol{\eta}, \boldsymbol{\mu}, \boldsymbol{\rho})=\sum_{i \in I}\left[f_{i}\left(\alpha_{i}\right)-g_{i}\left(\check{x}_{i}-\alpha_{i}\right)+\eta_{i}\left(\check{x}_{i}-\alpha_{i}\right)+\mu_{i}\left(\xi_{i}+y_{i}-\alpha_{i}\right)+\rho_{i} y_{i}\right]+\mu_{n e}\left(\xi_{n e}-\sum_{i \in I} y_{i}\right) .
$$

Note that since the constraints in the above optimization model are affine, the Slater's condition always hold (Boyd and Vandenberghe 2004). Let $\check{\boldsymbol{y}}$ and $\check{\boldsymbol{\alpha}}$ denote the optimal solution to Model (H.1), while $\check{\boldsymbol{\eta}}, \check{\boldsymbol{\mu}}$ and $\check{\boldsymbol{\rho}}$ are the optimal Lagrangian dual multipliers associated with the point $(\check{\boldsymbol{y}}, \check{\boldsymbol{\alpha}})$. Using the Langrangian duality we have:

$$
\frac{\partial Q}{\partial \xi_{i}}(\check{\boldsymbol{x}}, \boldsymbol{\xi}, \check{\boldsymbol{y}}, \check{\boldsymbol{\alpha}})=\frac{\partial L}{\partial \xi_{i}}(\check{\boldsymbol{x}}, \boldsymbol{\xi}, \check{\boldsymbol{y}}, \check{\boldsymbol{\alpha}})=\check{\mu}_{i}, \quad \text { and } \quad \frac{\partial Q}{\partial \xi_{n e}}(\check{\boldsymbol{x}}, \boldsymbol{\xi}, \check{\boldsymbol{y}}, \check{\boldsymbol{\alpha}})=\frac{\partial L}{\partial \xi_{n e}}(\check{\boldsymbol{x}}, \boldsymbol{\xi}, \check{\boldsymbol{y}}, \check{\boldsymbol{\alpha}})=\check{\mu}_{n e} .
$$

On the other hand, from the KKT conditions we have

$$
\begin{aligned}
\frac{\partial L}{\partial y_{i}}(\check{\boldsymbol{x}}, \boldsymbol{\xi}, \check{\boldsymbol{y}}, \check{\boldsymbol{\alpha}}) & =0 \Longrightarrow \check{\mu}_{i}+\check{\rho}_{i}-\check{\mu}_{n e}=0 \Longrightarrow \check{\mu}_{n e}=\check{\mu}_{i}+\check{\rho}_{i} \\
& \Longrightarrow \text { since } \rho_{i} \geq 0 \text { and consequently } \check{\rho}_{i} \geq 0 \text { for all } i \Longrightarrow \check{\mu}_{n e} \geq \check{\mu}_{i} \text { for all } i \text {. }
\end{aligned}
$$

From Equations (H.3) and (H.4), we can conclude that $\frac{\partial Q}{\partial \xi_{n e}}(\check{\boldsymbol{x}}, \boldsymbol{\xi}, \check{\boldsymbol{y}}, \check{\boldsymbol{\alpha}}) \geq \frac{\partial Q}{\partial \xi_{i}}(\check{\boldsymbol{x}}, \boldsymbol{\xi}, \check{\boldsymbol{y}}, \check{\boldsymbol{\alpha}})$ for all $i \in I$.

For Part 2, Equation (H.2) implies

$$
\frac{\partial Q}{\partial \alpha_{i}}(\check{\boldsymbol{x}}, \boldsymbol{\xi}, \check{\boldsymbol{y}}, \check{\boldsymbol{\alpha}})=0 \Longrightarrow f_{i}^{\prime}\left(\check{\alpha}_{i}\right)+g_{i}^{\prime}\left(\check{x}_{i}-\check{\alpha}_{i}\right)-\check{\eta}_{i}-\check{\mu}_{i}=0 .
$$

Consequently, from Equations (H.4) and (H.5) we have

$$
\check{\mu}_{n e}=f_{i}^{\prime}\left(\check{\alpha}_{i}\right)+g_{i}^{\prime}\left(\check{x}_{i}-\check{\alpha}_{i}\right)-\check{\eta}_{i}+\check{\rho}_{i}, \quad \text { for all } i \in I \text {. }
$$


Now, depending on the value of $\alpha_{i}$ at optimality, each delegation $i$ falls into one of the following sets.

$$
i \in\left\{\begin{array}{l}
V_{0}, \text { if } \check{\alpha}_{i}=\check{x}_{i} \leq \xi_{i}, \check{y}_{i}=0 \\
V_{1}, \text { if } \check{\alpha}_{i}=\check{y}_{i}+\xi_{i}=\check{x}_{i} \& \check{y}_{i}>0 \Longrightarrow \check{\rho}_{i}=0 \& \check{\eta}_{i}>0 \Longrightarrow \check{\mu}_{n e}<f_{i}^{\prime}\left(\check{\alpha}_{i}\right)+g_{i}^{\prime}\left(\check{x}_{i}-\check{\alpha}_{i}\right), \\
V_{2}, \text { if } \check{\alpha}_{i}=\check{y}_{i}+\xi_{i}<\check{x}_{i} \& \check{y}_{i}>0 \Longrightarrow \check{\rho}_{i}=0 \& \check{\eta}_{i}=0 \Longrightarrow \check{\mu}_{n e}=f_{i}^{\prime}\left(\check{\alpha}_{i}\right)+g_{i}^{\prime}\left(\check{x}_{i}-\check{\alpha}_{i}\right), \\
V_{3}, \text { if } \check{\alpha}_{i}=\check{y}_{i}+\xi_{i}<\check{x}_{i} \& \check{y}_{i}=0 \Longrightarrow \check{\rho}_{i}>0 \& \check{\eta}_{i}=0 \Longrightarrow \check{\mu}_{n e}>f_{i}^{\prime}\left(\check{\alpha}_{i}\right)+g_{i}^{\prime}\left(\check{x}_{i}-\check{\alpha}_{i}\right) .
\end{array}\right.
$$

According to Zipkin (1980) if we sort all $i$ in $V_{1} \cup V_{2} \cup V_{3}$, in terms of $f_{i}^{\prime}\left(\xi_{i}\right)+g_{i}^{\prime}\left(\check{x}_{i}-\xi_{i}\right)$ from highest to lowest, there will be an optimal separation level $k^{*}=\left|V_{1} \cup V_{2}\right|$ such that $\check{y}_{i}>0$ for $i=1, \ldots, k^{*}$ and $\check{y}_{i}=0$ for $i=k^{*}+1, \ldots, k^{*}+\left|V_{3}\right|$.

Now consider a problem with an additional delegation $j$, with $\check{x}_{j}$ and $\xi_{j}$, though the available resource, $\xi_{n e}$, stays the same. Denoting the optimal separation level of the new problem as $k^{* *}$, from Theorem 2 of Zipkin (1980), it can be deduced that $k^{* *} \geq k^{*}$. Moreover, from Theorem 1 of the same reference, we know that $\check{\mu}_{n e}$ is increasing in the separation level. Therefore, adding a new delegation does not decrease $\frac{\partial Q}{\partial \xi_{n e}}(\check{\boldsymbol{x}}, \boldsymbol{\xi}, \check{\boldsymbol{y}}, \check{\boldsymbol{\alpha}})=\check{\mu}_{n e}$. Note that if for the new delegation $j, \check{x}_{j}<\xi_{j}$, we will have $\check{y}_{j}=0$; consequently, the optimal allocation problem and $\frac{\partial Q}{\partial \xi_{n e}}(\check{\boldsymbol{x}}, \boldsymbol{\xi}, \check{\boldsymbol{y}}, \check{\boldsymbol{\alpha}})=\check{\mu}_{n e}$ remain unchanged. This ends the proof of Part 2.

For Part 3, from Equations (H.3), (H.5) and (H.6) we have

$$
\frac{\frac{\partial Q}{\partial \xi_{i}}(\check{\boldsymbol{x}}, \boldsymbol{\xi}, \check{\boldsymbol{y}}, \check{\boldsymbol{\alpha}})}{\frac{\partial Q}{\partial \xi_{n e}}(\check{\boldsymbol{x}}, \boldsymbol{\xi}, \check{\boldsymbol{y}}, \check{\boldsymbol{\alpha}})}=\frac{\check{\mu}_{i}}{\check{\mu}_{n e}}=\frac{f_{i}^{\prime}\left(\check{\alpha}_{i}\right)+g_{i}^{\prime}\left(\check{x}_{i}-\check{\alpha}_{i}\right)-\check{\eta}_{i}}{f_{i}^{\prime}\left(\check{\alpha}_{i}\right)+g_{i}^{\prime}\left(\check{x}_{i}-\check{\alpha}_{i}\right)-\check{\eta}_{i}+\check{\rho}_{i}}=1-\frac{\check{\rho}_{i}}{f_{i}^{\prime}\left(\check{\alpha}_{i}\right)+g_{i}^{\prime}\left(\check{x}_{i}-\check{\alpha}_{i}\right)-\check{\eta}_{i}+\check{\rho}_{i}}
$$

Let us refer to the value specified by Equation (H.8) as $A$. We have

$$
\frac{\partial A}{\partial a_{i}^{f}}=\frac{\partial f_{i}^{\prime}\left(\check{\alpha}_{i}\right) / \partial a_{i}^{f}}{\left[f_{i}^{\prime}\left(\check{\alpha}_{i}\right)+g_{i}^{\prime}\left(\check{x}_{i}-\check{\alpha}_{i}\right)-\check{\eta}_{i}+\check{\rho}_{i}\right]^{2}}>0 .
$$

The same holds true for $b_{i}^{f}$ and $a_{i}^{g}$. This ends the proof of Part 3.

Finally, for Part 4, we know from Part 1 that for every $\boldsymbol{x}$ and every possible realization of $\boldsymbol{\xi}=$ $\left[\tilde{\xi}_{1}, \ldots, \tilde{\xi}_{i}, \ldots, \tilde{\xi}_{n e}\right]$, we have

$$
Q\left(\boldsymbol{x},\left[\xi_{1}, \ldots, \xi_{i}+\delta, \ldots, \xi_{n e}\right]\right) \leq Q\left(\boldsymbol{x},\left[\xi_{1}, \ldots, \xi_{i}, \ldots, \xi_{n e}+\delta\right]\right)
$$

Therefore,

$$
\mathbb{E}_{\left[\tilde{\xi}_{1}, \ldots, \tilde{\xi}_{i}+\delta, \ldots, \tilde{\xi}_{n e}\right]}[Q(\boldsymbol{x}, \boldsymbol{\xi})] \leq \mathbb{E}_{\left[\tilde{\xi}_{1}, \ldots, \tilde{\xi}_{i}, \ldots, \tilde{\xi}_{n e}+\delta\right]}[Q(\boldsymbol{x}, \boldsymbol{\xi})], \quad \text { for all } i
$$

Since the above equation holds true for every $\boldsymbol{x}$, we have

$$
\mathfrak{Q}\left(\boldsymbol{x}^{*}\right)_{\tilde{\xi}_{i}+\delta} \leq \mathfrak{Q}^{*}\left(\boldsymbol{x}^{*}\right)_{\mid \tilde{\xi}_{n e}+\delta}, \quad \text { for all } i
$$

Proof of Proposition 2 To prove that $\boldsymbol{x}^{*}$ is increasing in $\boldsymbol{a}^{f}$, we first show that $\mathfrak{Q}(\boldsymbol{x})$ is supermodular in $\boldsymbol{x}$ and $\boldsymbol{a}^{f}$. For our discussions on supermodularity we refer to Simchi-Levi et al. (2014). First, it is straightforward to show that $U(\boldsymbol{x}, \boldsymbol{\xi}, \boldsymbol{y})$ is supermodular in $\boldsymbol{x}$ and $\boldsymbol{a}^{f}$, since $\frac{\partial^{2} U}{\partial \boldsymbol{x} \partial \boldsymbol{a}^{f}} \geq 0$. On the other hand, since $Q(\boldsymbol{x}, \boldsymbol{\xi})=\sup _{\boldsymbol{y}} U(\boldsymbol{x}, \boldsymbol{\xi}, \boldsymbol{y})$ and $U(\boldsymbol{x}, \boldsymbol{\xi}, \boldsymbol{y})$ is supermodular in $\boldsymbol{x}$ and $\boldsymbol{a}^{f}, Q(\boldsymbol{x}, \boldsymbol{\xi})$ is supermodular in $\boldsymbol{x}$ and $\boldsymbol{a}^{f}$ too. Finally, as $\mathfrak{Q}(\boldsymbol{x})=\mathbb{E}_{\tilde{\boldsymbol{\xi}}}[Q(\boldsymbol{x}, \boldsymbol{\xi})]$ is a linear combination of a supermodular function, $\mathfrak{Q}(\boldsymbol{x})$ is supermodular as well. 
Next, we show that $\mathfrak{Q}(\boldsymbol{x})$ is increasing in $\boldsymbol{a}^{f}>\mathbf{0}$. To that purpose, first note that $U(\boldsymbol{x}, \boldsymbol{\xi}, \boldsymbol{y})=$ $\sum_{i} u_{i}\left(x_{i}, \xi_{i}, y_{i}\right)$ is increasing in $\boldsymbol{a}^{f}$ for each $\boldsymbol{y}$. This implies that $Q(\boldsymbol{x}, \boldsymbol{\xi})$ is increasing in $\boldsymbol{a}^{f}$. Consequently, $\mathfrak{Q}(\boldsymbol{x})$ is increasing in $\boldsymbol{a}^{f}$.

Since $\mathfrak{Q}(\boldsymbol{x})$ is supermodular in $\boldsymbol{x}$ and $\boldsymbol{a}^{f}$, and the domain of $\boldsymbol{x}, \mathbb{R}_{+}^{n}$ is a lattice, according to Topkis's theorem (Topkis 2011), $\boldsymbol{x}^{*}=\arg \max _{\boldsymbol{x} \in \mathbb{R}_{+}^{n}} \mathfrak{Q}(\boldsymbol{x})$ is non-decreasing in $\boldsymbol{a}^{f}$. Similarly, one can prove the behavior of $\boldsymbol{x}^{*}$ in $\boldsymbol{a}^{g}$.

Proof of Proposition 3 We have $\alpha=\min \{\xi, x\}$. Therefore, Model (1) can be simplified to

$$
\mathfrak{Q}(x)=\max _{x} \int_{\xi=0}^{x}\left[f(\xi)-a^{g}(x-\xi)\right] B(\xi) \mathrm{d} \xi+\int_{\xi=x}^{\infty} f(x) B(\xi) \mathrm{d} \xi .
$$

Taking derivative of the above equation w.r.t. $x$ results in

$$
\begin{array}{r}
\frac{\partial \mathfrak{Q}(x)}{\partial x}=-a^{g} \int_{\xi=0}^{x} B(\xi) \mathrm{d} \xi+f(x) B(x)+\int_{\xi=x}^{\infty} f^{\prime}(x) B(\xi) \mathrm{d} \xi-f(x) B(x)=0 \\
\Longrightarrow f^{\prime}[1-\mathbb{B}(x)]=a^{g} \mathbb{B}(x) \Longrightarrow \mathbb{B}(x)=\frac{f^{\prime}(x)}{f^{\prime}(x)+a^{g}} .
\end{array}
$$

Proof of Proposition 4 The proof for the first inequality is straightforward. For the second inequality, let $\boldsymbol{x}^{*}$ be the solution that maximizes $\mathfrak{Q}(\boldsymbol{x})$; moreover, for a given $\boldsymbol{\xi}$, let $\overline{\boldsymbol{x}}(\boldsymbol{\xi})$ denote the solution which maximizes $Q(\boldsymbol{x}, \boldsymbol{\xi})$ for a given $\boldsymbol{\xi}$. That is

$$
\mathfrak{Q}\left(\boldsymbol{x}^{*}\right)=\max _{\boldsymbol{x} \geq \mathbf{0}} \mathfrak{Q}(\boldsymbol{x})=\max _{\boldsymbol{x} \geq \mathbf{0}} \mathbb{E}_{\tilde{\boldsymbol{\xi}}}[Q(\boldsymbol{x}, \boldsymbol{\xi})], \quad \text { and } \quad \mathbb{E}_{\tilde{\boldsymbol{\xi}}}\left[\max _{\boldsymbol{x} \geq \mathbf{0}} Q(\boldsymbol{x}, \boldsymbol{\xi})\right]=\mathbb{E}_{\tilde{\boldsymbol{\xi}}}[Q(\overline{\boldsymbol{x}}(\boldsymbol{\xi}), \boldsymbol{\xi})] .
$$

We have,

$$
\begin{aligned}
& Q\left(\boldsymbol{x}^{*}, \boldsymbol{\xi}\right) \leq Q(\overline{\boldsymbol{x}}(\boldsymbol{\xi}), \boldsymbol{\xi}) \Longrightarrow \mathbb{E}_{\tilde{\boldsymbol{\xi}}}\left[Q\left(\boldsymbol{x}^{*}, \boldsymbol{\xi}\right)\right] \leq \mathbb{E}_{\tilde{\boldsymbol{\xi}}}[Q(\overline{\boldsymbol{x}}(\boldsymbol{\xi}), \boldsymbol{\xi})], \quad \forall \boldsymbol{\xi} \in \mathbb{R}_{n+1}^{+}, \\
& \Longrightarrow \mathfrak{Q}\left(\boldsymbol{x}^{*}\right)=\max _{\boldsymbol{x} \geq \mathbf{0}} \mathbb{E}_{\tilde{\boldsymbol{\xi}}}[Q(\boldsymbol{x}, \boldsymbol{\xi})] \leq \mathbb{E}_{\tilde{\boldsymbol{\xi}}}\left[\max _{\boldsymbol{x} \geq \mathbf{0}} Q(\boldsymbol{x}, \boldsymbol{\xi})\right] .
\end{aligned}
$$

For the last inequality, from Part 4 of Lemma 3, we know that $Q(\boldsymbol{x}, \boldsymbol{\xi})$ is jointly concave in $(\boldsymbol{x}, \boldsymbol{\xi})$. Now, we show that $\max _{\boldsymbol{x}} Q(\boldsymbol{x}, \boldsymbol{\xi})$ is a concave function of $\boldsymbol{\xi}$. Assume $T(\boldsymbol{\xi})=\max _{\boldsymbol{x}} Q(\boldsymbol{x}, \boldsymbol{\xi})$. Moreover, let $\boldsymbol{x}^{1}$ and $\boldsymbol{x}^{2}$ represent the optimal solutions of $T\left(\boldsymbol{\xi}^{1}\right)$ and $T\left(\boldsymbol{\xi}^{2}\right)$. We have

$$
\begin{aligned}
\lambda T\left(\boldsymbol{\xi}^{1}\right)+(1-\lambda) T\left(\boldsymbol{\xi}^{2}\right) & =\lambda Q\left(\boldsymbol{x}^{1}, \boldsymbol{\xi}^{1}\right)+(1-\lambda) Q\left(\boldsymbol{x}^{2}, \boldsymbol{\xi}^{2}\right) \leq Q\left(\lambda\left(\boldsymbol{x}^{1}, \boldsymbol{\xi}^{1}\right)+(1-\lambda)\left(\boldsymbol{x}^{2}, \boldsymbol{\xi}^{2}\right)\right) \\
& \leq \max _{\boldsymbol{x} \geq \mathbf{0}} Q\left(\boldsymbol{x}, \lambda \boldsymbol{\xi}^{1}+(1-\lambda) \boldsymbol{\xi}^{2}\right)=T\left(\lambda \boldsymbol{\xi}^{1}+(1-\lambda) \boldsymbol{\xi}^{2}\right) .
\end{aligned}
$$

Therefore $\max _{\boldsymbol{x} \geq \mathbf{0}} Q(\boldsymbol{x}, \boldsymbol{\xi})$ is concave in $\boldsymbol{\xi}$. Using Jensen's inequality, it follows that $\mathbb{E}_{\tilde{\boldsymbol{\xi}}}\left[\max _{\boldsymbol{x} \geq \mathbf{0}} Q(\boldsymbol{x}, \boldsymbol{\xi})\right] \leq$ $\max _{\boldsymbol{x} \geq \mathbf{0}} Q(\boldsymbol{x}, \overline{\boldsymbol{\xi}})=Q(\overline{\boldsymbol{x}}, \overline{\boldsymbol{\xi}})$, which is the desired third inequality.

Proof of Lemma 2 The objective function is concave and separable on each variable. Moreover, the constraints are linear and form a convex set; therefore, $\overline{\mathfrak{Q}}$ is jointly concave in $(\boldsymbol{x}, \boldsymbol{\alpha}, \boldsymbol{y})$.

Proof of Theorem 1 For a given $\check{\boldsymbol{x}}$, we can rewrite the $k$-th Benders subproblem $Q\left(\check{\boldsymbol{x}}, \boldsymbol{\xi}^{k}\right)$ as follows, assuming $u_{i}\left(\check{x}_{i}, \xi_{i}^{k}, y_{i}^{k}\right)=f_{i}\left(\xi_{i}^{k}+y_{i}^{k}\right)-g_{i}\left(\check{x}_{i}-\xi_{i}^{k}+y_{i}^{k}\right)$, and $\bar{y}_{i}^{k}=\check{x}_{i}-\xi_{i}^{k}$.

$$
\begin{array}{ll}
\max _{\boldsymbol{y}^{k}} & \sum_{i \in \mathfrak{C}\left(\check{\boldsymbol{x}}, \boldsymbol{\xi}^{k}\right)} u_{i}\left(\check{x}_{i}, \xi_{i}^{k}, y_{i}^{k}\right)+\sum_{i \notin \mathfrak{C}\left(\check{\boldsymbol{x}}, \boldsymbol{\xi}^{k}\right)} f_{i}\left(x_{i}\right), \\
\text { s.t. } & \sum_{i \in \mathfrak{C}\left(\check{\boldsymbol{x}}, \boldsymbol{\xi}^{k}\right)} y_{i}^{k} \leq \xi_{n e}, \\
& y_{i}^{k} \leq \bar{y}_{i}^{k}, \quad i \in \mathfrak{C}\left(\check{\boldsymbol{x}}, \boldsymbol{\xi}^{k}\right) \\
& y_{i}^{k} \geq 0, \quad i \in I
\end{array}
$$


Let $\mu_{n e}^{k}, \mu_{i}^{k}$, and $\rho_{i}^{k}$ refer to the dual variables associated with the first, second and third constraints, respectively. The associated Lagrangian function reads as:

$$
\begin{aligned}
L^{k}(\check{\boldsymbol{x}}, \boldsymbol{\mu}, \boldsymbol{\rho})= & \sum_{i \in \mathfrak{C}\left(\check{\boldsymbol{x}}, \boldsymbol{\xi}^{k}\right)}\left[u_{i}\left(\check{x}_{i}, \xi_{i}^{k}, y_{i}^{k}\right)+\mu_{i}^{k}\left(\check{x}_{i}-\xi_{i}^{k}-y_{i}^{k}\right)\right]+\mu_{n e}^{k}\left(\xi_{n e}^{k}-\sum_{i \in \mathfrak{C}\left(\check{\boldsymbol{x}}, \boldsymbol{\xi}^{k}\right)} y_{i}^{k}\right) \\
& +\sum_{i \notin \mathfrak{C}\left(\check{\boldsymbol{x}}, \boldsymbol{\xi}^{k}\right)} f_{i}\left(\check{x}_{i}\right)+\sum_{i \in I} \rho_{i}^{k} y_{i}^{k} .
\end{aligned}
$$

It is not difficult to see that for $\boldsymbol{\lambda}^{k}$ given in (10) we have:

$$
\lambda_{i}^{k} \in \frac{\partial L^{k}}{\partial x_{i}}(\check{\boldsymbol{x}}, \check{\boldsymbol{\mu}}, \check{\boldsymbol{\rho}}), \quad i \in I, k \in K
$$

where $\check{\boldsymbol{\mu}}, \check{\boldsymbol{\rho}}$ are the optimal Lagrangian dual multipliers associated with the point $\check{\boldsymbol{y}}$. Hence, $\boldsymbol{\lambda}^{k}$ calculated as in (10) gives a valid supergradient vector for $Q\left(\boldsymbol{x}, \boldsymbol{\xi}^{k}\right)$. It remains to show that the values of $\boldsymbol{\mu}$ given in (11)-(12) are properly calculated. Using the KKT conditions for the Lagrangian function, we have:

$$
\begin{array}{ll}
\frac{\partial L^{k}}{\partial y_{i}^{k}}=\frac{\partial u_{i}}{\partial y_{i}}\left(\check{x}_{i}, \xi_{i}^{k}, \check{y}_{i}^{k}\right)-\mu_{n e}^{k}-\mu_{i}^{k}+\rho_{i}^{k}=0, & i \in \mathfrak{C}\left(\check{\boldsymbol{x}}, \boldsymbol{\xi}^{k}\right), \\
\rho_{i}^{k} y_{i}^{k}=0, & i \in I, \\
\mu_{n e}^{k} \geq 0, \rho_{i}^{k}, \mu_{i}^{k} \geq 0, & i \in I .
\end{array}
$$

Hence, we have

$$
\text { if } \check{y}_{i}^{k}>0 \Longrightarrow \check{\rho}_{i}^{k}=0 \Leftrightarrow \check{\mu}_{i}^{k}=\frac{\partial u_{i}}{\partial y_{i}}\left(\check{x}_{i}, \xi_{i}^{k}, \check{y}_{i}^{k}\right)-\check{\mu}_{n e}^{k} .
$$

From complementary slackness we also have $\check{\mu}_{i}^{k}\left(\bar{y}_{i}^{k}-\check{y}_{i}^{k}\right)=0$; therefore

$$
0 \leq \check{y}_{i}^{k}<\bar{y}_{i}^{k} \Longrightarrow \check{\mu}_{i}^{k}=0 .
$$

Moreover, from Equations (H.10) and (H.11) it follows:

$$
\text { if } 0<\check{y}_{i}^{k}<\bar{y}_{i}^{k} \Longrightarrow \check{\mu}_{i}^{k}=\frac{\partial u_{i}}{\partial y_{i}}\left(\check{x}_{i}, \xi_{i}^{k}, \check{y}_{i}^{k}\right)-\check{\mu}_{n e}^{k}=0 \Leftrightarrow \check{\mu}_{n e}^{k}=\frac{\partial u_{i}}{\partial y_{i}}\left(\check{x}_{i}, \xi_{i}^{k}, \check{y}_{i}^{k}\right) \text {. }
$$

Note that the latter result implies that the values of $\frac{\partial u_{i}}{\partial y_{i}}\left(\check{x}_{i}, \xi_{i}^{k}, \check{y}_{i}^{k}\right)$ are the same for all $i$ such that $0<\check{y}_{i}^{k}<\bar{y}_{i}^{k}$, which is why any such $j \in I$ can be used to obtain $\check{\mu}_{n e}^{k}$ in (12). This ends the proof.

Proof of Corollary ?? Let us refer to the space of feasible solutions of $Q, Q^{\prime}$ and $Q^{\prime \prime}$ as $C, C^{\prime}$ and $C^{\prime \prime}$ respectively. $C \subset C^{\prime}$, since the difference between the two is the right hand side of the budget allocation constraint, and $\xi_{n e} \leq \xi_{n e}+\sum_{i}\left[\xi_{i}-x_{i}\right]^{+}$. On the other hand:

$$
\begin{aligned}
& C^{\prime}=\left\{\sum_{i} y_{i} \leq \xi_{n e}+\sum_{i}\left[\xi_{i}-x_{i}\right]^{+} ; \boldsymbol{y} \geq 0\right\}, \\
& C^{\prime \prime}=\left\{\sum_{i} y_{i} \leq \xi_{n e} ; y_{i} \geq-\xi_{i} \forall i\right\} \equiv\left\{\sum_{i} y_{i} \leq \xi_{n e}+\sum_{i} \xi_{i} ; \boldsymbol{y} \geq 0\right\}, \\
& \Longrightarrow \forall \boldsymbol{y} \in C^{\prime} \rightarrow \boldsymbol{y} \in C^{\prime \prime} \Longrightarrow C^{\prime} \subset C^{\prime \prime}
\end{aligned}
$$

As the objective function remains the same in all three problems, we can conclude that $\mathfrak{Q}\left(\boldsymbol{x}^{*}\right) \leq$ $\max _{\boldsymbol{x}} \mathbb{E}_{\tilde{\boldsymbol{\xi}}}\left[Q^{\prime}(\boldsymbol{x}, \boldsymbol{\xi})\right] \leq \max _{\boldsymbol{x}} \mathbb{E}_{\tilde{\boldsymbol{\xi}}}\left[Q^{\prime \prime}(\boldsymbol{x}, \boldsymbol{\xi})\right]$.

The proof also holds for the model with target-dependent donations. Any feasible $\boldsymbol{x}$ for $\mathscr{Q}(\boldsymbol{x})$, including $\arg \max \mathscr{Q}(\boldsymbol{x})$ is also feasible for $\mathbb{E}_{\tilde{\boldsymbol{\xi}} \mid \boldsymbol{x}}\left[Q^{\prime}(\boldsymbol{x}, \boldsymbol{\xi})\right]$. On the other hand, for any given $\boldsymbol{x}$ and $\boldsymbol{\xi}$, the space of feasible solutions, i.e., $\boldsymbol{y}$ for $Q(\boldsymbol{x}, \boldsymbol{\xi})$ is a subset of that for $Q^{\prime}(\boldsymbol{x}, \boldsymbol{\xi})$. Thus, $\mathscr{Q}\left(\boldsymbol{x}^{*}\right) \leq \max _{\boldsymbol{x} \geq \mathbf{0}} \mathbb{E}_{\tilde{\boldsymbol{\xi}} \mid \boldsymbol{x}}\left[Q^{\prime}(\boldsymbol{x}, \boldsymbol{\xi})\right]$. The same reasoning applies to the second inequality. 
Proof of Lemma 3 First, note that the linearity constraint qualification holds for $Q(\boldsymbol{x}, \boldsymbol{\xi})$.

Part 1: The function $\min \left\{x_{i}, \xi_{i}+y_{i}\right\}$ is concave in $y_{i}$. Since $f_{i}$ is concave in its argument, $f_{i}\left(\min \left\{x_{i}, \xi_{i}+y_{i}\right\}\right)$ is also concave in $y_{i}$. Similarly, $\left[x_{i}-\xi_{i}-y_{i}\right]^{+}=\max \left\{0, x_{i}-\xi_{i}-y_{i}\right\}$ is convex in $y_{i}$. Since $g_{i}$ is convex in its argument, $g_{i}\left(\left[x_{i}-\xi_{i}-y_{i}\right]^{+}\right)$is also convex in $y_{i}$, and therefore $u_{i}\left(x_{i}, \xi_{i}, y_{i}\right)=f_{i}\left(\min \left\{x_{i}, \xi_{i}+y_{i}\right\}\right)-g_{i}\left(\left[x_{i}-\right.\right.$ $\left.\left.\xi_{i}-y_{i}\right]^{+}\right)$is concave in $y_{i}$. As a result, $\sum_{i \in \mathfrak{C}(\boldsymbol{x}, \boldsymbol{\xi})}\left[f_{i}\left(\xi_{i}+y_{i}\right)-g_{i}\left(\left[x_{i}-\xi_{i}-y_{i}\right]^{+}\right)\right]$is concave in $\boldsymbol{y}$. Note that $\sum_{i \notin \mathfrak{C}(\boldsymbol{x}, \boldsymbol{\xi})} f_{i}\left(x_{i}\right)$ is a constant. Since the constraints form a convex set, $\sum_{i=1}^{n} u_{i}\left(x_{i}, \xi_{i}, y_{i}\right)$ is concave in $\boldsymbol{y}$. Moreover, it is easy to show that for all $i \in \mathfrak{C}(\boldsymbol{x}, \boldsymbol{\xi}), u_{i}\left(x_{i}, \xi_{i}, y_{i}\right)=f_{i}\left(\xi_{i}+y_{i}\right)-g_{i}\left(x_{i}-\xi_{i}-y_{i}\right)$ is non-decreasing in $y_{i}$ and therefore, $\sum_{i=1}^{n} u_{i}\left(x_{i}, \xi_{i}, y_{i}\right)$ is non-decreasing in $\boldsymbol{y}$.

Part 2: Similar to Birge and Louveaux (2011), we define

$$
\begin{array}{ll} 
& K_{1}=\left\{\boldsymbol{x} \in \mathbb{R}^{n}: \boldsymbol{x} \geq 0\right\} \\
& K_{2}(\boldsymbol{\xi})=\left\{\boldsymbol{x} \in \mathbb{R}^{n}: \exists \boldsymbol{y}(\boldsymbol{\xi}) \mid \boldsymbol{y} \geq \mathbf{0} \text { and } \sum_{i=1}^{n} y_{i} \leq \xi_{n e}\right\} \\
\text { and } \quad & K_{2}=\left\{\boldsymbol{x} \in \mathbb{R}^{n}: \mathfrak{Q}(\boldsymbol{x})>-\infty\right\}
\end{array}
$$

where $K_{1}$ refers to the feasible set of Stage 1 of Model (1), $K_{2}$ refers to the values of $\boldsymbol{x}$ for which the recourse problem is feasible (independently of the realization of random elements in the second stage), and $K_{2}(\boldsymbol{\xi})$ refers to the values of $\boldsymbol{x}$ for which the recourse problem is feasible for a given realization of $\boldsymbol{\xi}$. Since $\boldsymbol{x}$ does not appear in the constraints of the recourse problem, every feasible value of $\boldsymbol{x}$ in Model (1) is also feasible in Model (2), i.e., $K_{1} \subset K_{2}$ (indeed $K_{1}=K_{2}$ ), and therefore, the stochastic program has relatively complete recourse. On the other hand, regardless of choice of $\boldsymbol{x}$ and realization of $\boldsymbol{\xi} \in \mathbb{R}_{\geq 0}^{n+1}$, there exist a recourse action $\boldsymbol{y}=0$, thus, the problem has a complete recourse as well.

Part 3: $u_{i}\left(x_{i}, \xi_{i}, y_{i}\right)$ is non-decreasing in $\xi_{i}$ for all $i$; moreover, $\boldsymbol{y}$ is non-decreasing in $\xi_{n e}$, i.e., $y_{i}$ is nondecreasing in $\xi_{n e}$ for all $i \in I$. Therefore, $U(\boldsymbol{x}, \boldsymbol{\xi}, \boldsymbol{y})$ is non-decreasing in $\boldsymbol{\xi}$ for any given $\boldsymbol{x}$. Consequently, $Q(\boldsymbol{x}, \boldsymbol{\xi})=\max _{\boldsymbol{y} \geq \mathbf{0}} U(\boldsymbol{x}, \boldsymbol{\xi}, \boldsymbol{y})$ is non-decreasing in $\boldsymbol{\xi}$.

The proofs of Parts 4 to 6 are similar to (Birge and Louveaux 2011). Thus we skip these proofs for conciseness. 